\title{
Conversion of Aryl Azides to O-Alkyl Imidates Via Modified Staudinger Ligation
}

José A. Restituyo, Lindsay R. Comstock, Scott G. Petersen, Thomas Stringfellow and Scott R. Rajski

General: All reactions were carried out under an inert atmosphere of argon unless indicated otherwise. All reagents were obtained from available commercial sources and used without additional purification unless otherwise noted. Anhydrous THF was from a J.T. Baker Cycle-Tainer product number 9446-Q1. NMR spectra were recorded on Varian Unity Inova $400 \mathrm{MHz}$ and $500 \mathrm{MHz}$ spectrometers using either TMS or solvent as the internal reference; the chemical shifts are reported in ppm, in $\delta$ units. High resolution mass spectral data were obtained using an IonSpec HiResMALDI FT-Mass Spectrometer with a 7 tesla superconducting magnet. HPLC mass spectral data were obtained using an Agilent 1100 HPLC-MSD SL quadrupole mass spectrometer. HPLC chromatograms disclosed were obtained using a Waters 600E 4-Solvent delivery system with 717 Plus autosampler with heater/cooler, 4 channel in-line degasser, and Millenium 3.2 (M32) software package. All solvents were $0.1 \%$ in TFA and HPLC grade quality. All reactions analyzed by HPLC were run in a $1: 1$ mixture of THF: $\mathrm{H}_{2} \mathrm{O}$ at $30^{\circ} \mathrm{C}$ for the noted period of time and were $\sim 1 \mathrm{mM}$ in both coupling components.

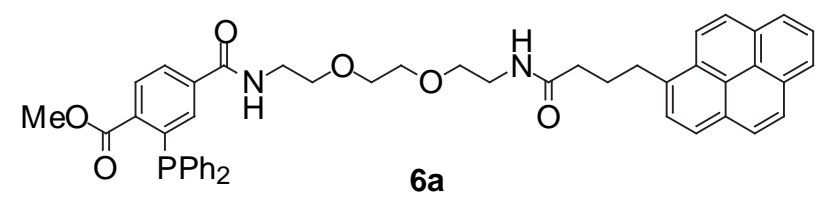

\section{2-Diphenylphosphanyl-N-(2-\{2-[2-(4-pyren-1-yl-butyrylamino)-ethoxy]-ethoxy\}- ethyl)-terephthalamic acid methyl ester (6a).}

To $250 \mathrm{mg}$ of methyl 2-(diphenylphosphanyl) benzoic acid $(0.687 \mathrm{mmol})$ was added 3.4 mL of a 2:1:1 mixture of THF:DMF: $\mathrm{H}_{2} \mathrm{O}$ along with $343 \mu \mathrm{L}$ distilled

diisopropylethylamine (DIEA) $(2 \mathrm{mmol})$. The solution was stirred at ambient temperature for $5 \mathrm{~min}$ followed by addition of $260 \mathrm{mg}(0.860 \mathrm{mmol}) \mathrm{O}-(\mathrm{N}-\mathrm{Succinimidyl})-\mathrm{N}, \mathrm{N}, \mathrm{N}^{\prime}, \mathrm{N}^{\prime}-$ tetramethyluronium tetrafluoroborate (TSTU). The reaction was stirred $1 \mathrm{~h}$ at ambient temperature followed by dilution with $2.5 \mathrm{~mL}$ anhydrous THF and transferred to a stirring solution of $1 \mathrm{~mL} 2,2^{\prime}$-(Ethylenedioxy) diethylamine $(6.8 \mathrm{mmol})$ diluted in $3 \mathrm{~mL}$ anhydrous THF. The reaction was stirred $2 \mathrm{~h}$ at room temperature and then partitioned between saturated $\mathrm{NaHCO}_{3}(\mathrm{pH}=9.5)$ and $\mathrm{CHCl}_{3}$. The layers were separated and the aqueous fraction extracted twice with $20 \mathrm{~mL} \mathrm{CHCl}_{3}$. The organic layers were then combined and washed with saturated $\mathrm{NaHCO}_{3}(3 \times 30 \mathrm{~mL})$ which was sufficient for removal of excess diamine. $0.3 \mathrm{mmol}$ of this crude material was then coupled to the activated NHS ester of pyrenebutyric acid in the following manner. To $86.5 \mathrm{mg}(0.3 \mathrm{mmol})$ pyrenebutyric acid was added $1.5 \mathrm{~mL}$ of the THF:DMF: $\mathrm{H}_{2} \mathrm{O}$ mixture noted above along with $150 \mathrm{~mL}$ $(.87 \mathrm{mmol})$ DIEA. The solution of acid was stirred $5 \mathrm{~min}$ at ambient temperature followed by addition of $113 \mathrm{mg}$ TSTU $(0.37 \mathrm{mmol})$. The NHS ester was formed over the course of $1 \mathrm{~h}$ at room temperature and the activated ester diluted with $1 \mathrm{~mL}$ THF. To the NHS ester was then added $0.3 \mathrm{mmol}$ of the phosphanyl amine diluted into $1.2 \mathrm{~mL}$ anhydrous THF. The coupling was performed for $2 \mathrm{~h}$ at room temperature, the reaction then partitioned between $\mathrm{CHCl}_{3}$ and cold, saturated $\mathrm{NaHCO}_{3}$. Layers were separated and then the $\mathrm{CHCl}_{3}$ layer washed with saturated $\mathrm{NaHCO}_{3}(2 \times 30 \mathrm{~mL}), 0.5 \mathrm{~N} \mathrm{HCl}(3 \times 50 \mathrm{~mL}), \mathrm{H}_{2} \mathrm{O}(2 \mathrm{x}$ 
$50 \mathrm{~mL})$, and brine $(2 \times 20 \mathrm{~mL})$. Finally the solution was dried over $\mathrm{Na}_{2} \mathrm{SO}_{4}$, solvents removed in vacuo and the coupled material subjected to 2 rounds of PTLC purification (5:5:3:1 Hex: $\mathrm{CH}_{2} \mathrm{Cl}_{2}: \mathrm{EtOAc:MeOH}$ ) to afford $97.5 \mathrm{mg} \mathbf{6 a}$ (42.5\% overall yield).

${ }^{1} \mathrm{H} \mathrm{NMR}\left(\mathrm{CDCl}_{3}\right) \delta 8.30(\mathrm{~d}, \mathrm{~J}=9.2 \mathrm{~Hz}, 1 \mathrm{H}), 8.16(\mathrm{~d}, \mathrm{~J}=7.6 \mathrm{~Hz}, 2 \mathrm{H}), 8.10(\mathrm{~d}, \mathrm{~J}=9.2 \mathrm{~Hz}$, $2 \mathrm{H}), 8.05-7.96(\mathrm{~m}, 4 \mathrm{H}), 7.85(\mathrm{~d}, \mathrm{~J}=7.6 \mathrm{~Hz}, 1 \mathrm{H}), 7.71(\mathrm{dd}, \mathrm{J}=8.0,1.6 \mathrm{~Hz}, 1 \mathrm{H}), 7.39-7.25$ $(\mathrm{m}, 10 \mathrm{H}), 6.34-6.26(\mathrm{~m}, 1 \mathrm{H}), 5.96-5.88(\mathrm{~m}, 1 \mathrm{H}), 3.77(\mathrm{~s}, 3 \mathrm{H}), 3.60-3.32(\mathrm{~m}, 14 \mathrm{H}), 2.32-$ $2.12(\mathrm{~m}, 4 \mathrm{H}) .{ }^{13} \mathrm{C} \mathrm{NMR}\left(\mathrm{CDCl}_{3}\right) \delta 173.0,166.9,166.7,141.9,141.6,137.5,137.4,136.8$, 136.1, 134.2, 134.0, 133.0, 131.6, 131.1, 131.0, 130.1, 129.2, 129.0, 128.8, 127.7, 127.6, $127.0,126.8,126.1,125.2,125.1,125.0,123.6,70.4,70.3,70.1,69.7,68.2,52.4,50.9$, 40.0, 39.4,36.2, 33.0, 27.6, 25.8. HRMS (MALDI): calcd for $\mathrm{C}_{47} \mathrm{H}_{45} \mathrm{~N}_{2} \mathrm{O}_{6} \mathrm{P}[\mathrm{M}+\mathrm{Na}]=$ 787.2913, measured 787.2887.

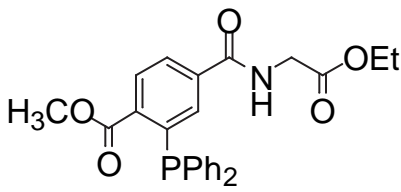

$6 b$

\section{2-Diphenylphosphanyl-N-ethoxycarbonylmethyl-terephthalamic acid methyl ester} (6b).

To $150 \mathrm{mg}$. of methyl 2-(diphenylphosphanyl) benzoic acid $(0.42 \mathrm{mmol})$ was added $2 \mathrm{~mL}$ of a 2:1:1 mixture of THF:DMF: $\mathrm{H}_{2} \mathrm{O}$ along with $206 \mu \mathrm{L}$ diisopropylethylamine (DIEA)(1.2 mmol). The solution was stirred at ambient temperature for $5 \mathrm{~min}$ followed by addition of $260 \mathrm{mg}(0.516 \mathrm{mmol}) \mathrm{O}-(\mathrm{N}-\mathrm{Succinimidyl})-\mathrm{N}, \mathrm{N}, \mathrm{N}^{\prime}, \mathrm{N}^{\prime}$-tetramethyluronium tetrafluoroborate (TSTU). The reaction was stirred $30 \mathrm{~min}$ at ambient temperature followed addition of $430 \mu \mathrm{L}$ DIEA $(2.52 \mathrm{mmol})$ and $172.5 \mathrm{mg}(1.26 \mathrm{mmol})$ glycine ethyl ester hydrochloride. The reaction was stirred $4 \mathrm{~h}$ at room temperature and then partitioned between $20 \mathrm{~mL}$ cold $1 \mathrm{~N} \mathrm{HCl}$ and $\mathrm{CHCl}_{3}(30 \mathrm{~mL})$. Layers were separated and the organic fraction washed 3 more times with $20 \mathrm{~mL}$ cold $1 \mathrm{~N} \mathrm{HCl}$ then 2 times with saturated $\mathrm{NaHCO}_{3}(2 \times 20 \mathrm{~mL})$ and brine. Solvent was dried over $\mathrm{Na}_{2} \mathrm{SO}_{4}$ followed by filtration of solids and removal of solvent in vacuo. Column chromatography (10:10:6:2:1, Hex: $\mathrm{CH}_{2} \mathrm{Cl}_{2}$ :EtOAc:MeOH:Pet Et) afforded $79.4 \mathrm{mg}$ 6b (43\% yield) ${ }^{1} \mathrm{H} \mathrm{NMR}\left(\mathrm{CDCl}_{3}\right) \delta 8.09(\mathrm{dd}, \mathrm{J}=8.1,3.5 \mathrm{~Hz}, 1 \mathrm{H}), 7.81(\mathrm{dd}, \mathrm{J}=8.1,1.5 \mathrm{~Hz}, 1 \mathrm{H}), 7.38-$ $7.22(\mathrm{~m}, 11 \mathrm{H}), 6.45-6.38(\mathrm{~m}, 1 \mathrm{H}), 4.2$ (q, J=7.2 Hz, 2H), 4.08 (d, J=5.2 Hz, 2H), 3.74 (s, $3 \mathrm{H}), 1.27(\mathrm{t}, \mathrm{J}=7.2 \mathrm{~Hz}, 3 \mathrm{H}) ;{ }^{13} \mathrm{C} \mathrm{NMR}\left(\mathrm{CDCl}_{3}\right) \delta 169.8,166.8,166.4,142.1,141.8$, 137.2, 136.6, 134.2, 133.9, 132.7, 131.2, 129.3, 129.0, 128.9, 127.1, 61.9, 52.5, 42.1, 14.4. HRMS (MALDI): calcd for $\mathrm{C}_{25} \mathrm{H}_{24} \mathrm{NO}_{6} \mathrm{P}[\mathrm{M}+\mathrm{Na}]=472.1290$, measured 472.1296. 


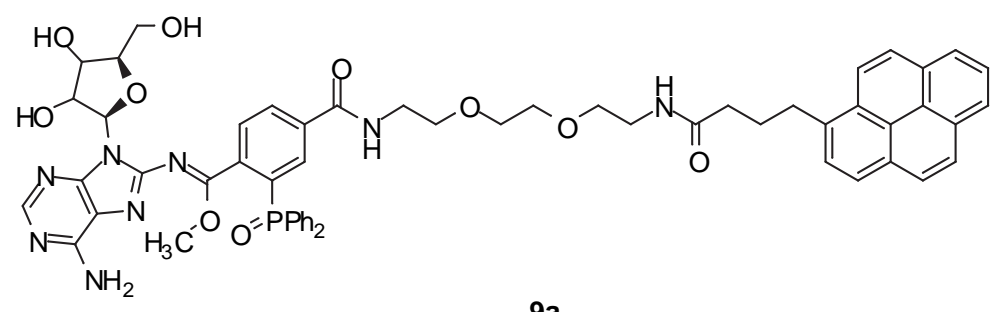

(Z)-methyl N-6-amino-9-((2R,5S)-3,4-dihydroxy-5-(hydroxymethyl)-tetrahydrofuran-2-yl)-9H-purin-8-yl-2-(diphenylphosphoryl)-4-((2-(2-(2-(4-pyren-1yl)butanamido)ethoxy)ethoxy)ethyl)carbamoyl)benzimidate (9a). To 8-

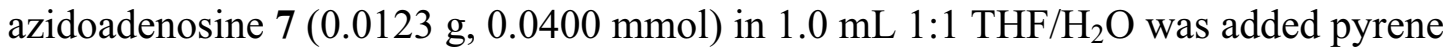

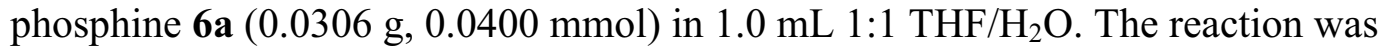
incubated at $30{ }^{\circ} \mathrm{C}$ for $2 \mathrm{~h}$. The reaction mixture was extracted with ethyl acetate, washed with brine, and dried over $\mathrm{Na}_{2} \mathrm{SO}_{4}$. The solvent was removed and the resulting material purified by flash chromatography [3:2 EtOAc:Hex.] to yield 9a as a light yellowish oil (34 mg, 82\% yield). TLC and HPLC-MS revealed this material to be identical to that identified during the course of HPLC timetrial analyses. ${ }^{1} \mathrm{H} \mathrm{NMR}\left(\mathrm{CDCl}_{3}\right) \delta 8.37(\mathrm{~d}, \mathrm{~J}=$ $14.4 \mathrm{~Hz}, 1 \mathrm{H}), 8.24$ (d, J=9.6 Hz, 1H), 8.14-7.94 (m, 9H), 7.87-7.80 (m, 5H), 7.53-7.42 $(\mathrm{m}, 5 \mathrm{H}), 6.56(\mathrm{~d}, \mathrm{~J}=7.2 \mathrm{~Hz}, 1 \mathrm{H}), 5.01(\mathrm{dd}, \mathrm{J}=6.8,5.2 \mathrm{~Hz}, 1 \mathrm{H}), 4.48(\mathrm{~d}, \mathrm{~J}=5.2 \mathrm{~Hz}, 1 \mathrm{H})$, $4.30(\mathrm{~s}, 1 \mathrm{H}), 3.96(\mathrm{~d}, \mathrm{~J}=12.8 \mathrm{~Hz}, 1 \mathrm{H}), 3.73-3.25(\mathrm{~m}, 27 \mathrm{H}), 2.27-2.01(\mathrm{~m}, 4 \mathrm{H}) .{ }^{13} \mathrm{C} \mathrm{NMR}$ $\left(\mathrm{CDCl}_{3}\right) \delta 173.01,167.75,166.38,154.75,151.58,148.67,147.89,135.70,135.61$, $132.72,130.75,130.66,131.81,128.95,128.55,126.68,126.30,125.68,125.05,124.63$, $124.15,120.62,70.42,70.36,70.15,69.79,68.20,52.44,50.97,40.01,39.42,36.22$, 33.05, 27.66, 25.87. ${ }^{31} \mathrm{P} \mathrm{NMR}\left(\mathrm{CDCl}_{3}\right) \delta 22.6$. HRMS (MALDI): calcd for $\mathrm{C}_{357} \mathrm{H}_{57} \mathrm{~N}_{8}$ $\mathrm{O}_{10} \mathrm{P}[\mathrm{M}+\mathrm{Na}]=1067.3833$, measured 1067.3420 .

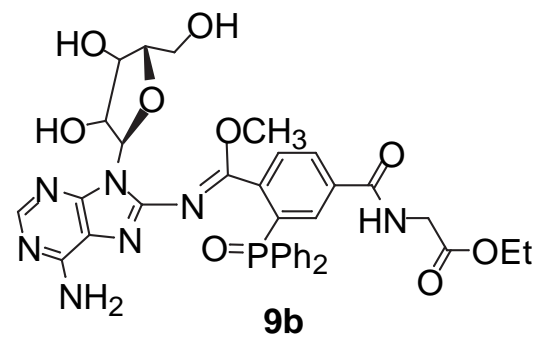

[4-[6-Amino-9-(3,4-dihydroxy-5-hydroxymethyl-tetrahydro-furan-2-yl)-9H-purin-8ylcarbamoyl]-3-(diphenyl-phosphinoyl)-benzoylamino]-acetic acid ethyl ester (9b). To 8-azidoadenosine $7(0.0123 \mathrm{~g}, 0.0400 \mathrm{mmol})$ in $1.0 \mathrm{~mL} 1: 1 \mathrm{THF} / \mathrm{H}_{2} \mathrm{O}$ was added the glycine phosphine $6 \mathbf{b}(0.0174 \mathrm{~g}, 0.0400 \mathrm{mmol})$ in $1.0 \mathrm{~mL} 1: 1 \mathrm{THF} / \mathrm{H}_{2} \mathrm{O}$ and reaction stirred at $30{ }^{\circ} \mathrm{C}$ for $2 \mathrm{~h}$. The reaction mixture was extracted with ethyl acetate, washed with brine, and dried over $\mathrm{Na}_{2} \mathrm{SO}_{4}$. The solvent was removed and the resulting material was purified by flash chromatography [3:2 EtOAc:Hex.] to yield $\mathbf{9 b}$ as a yellowish oil (24 mg, 84\% yield). As with 9a, this material was identical in HPLC retention time and mass spectroscopic analysis to that material identified during HPLC timetrial analyses. The independently purified substances were also identical in $\mathrm{R}_{\mathrm{f}}$. 
${ }^{1} \mathrm{H}$ NMR $\left(\mathrm{CDCl}_{3}\right) \delta 8.28(\mathrm{~d}, \mathrm{~J}=14.4 \mathrm{~Hz}, 1 \mathrm{H}), 8.04(\mathrm{~d}, \mathrm{~J}=8 \mathrm{~Hz}, 1 \mathrm{H}), 7.87-7.77(\mathrm{~m}, 5 \mathrm{H})$, $7.73(\mathrm{~s}, 1 \mathrm{H}), 7.52-7.39(\mathrm{~m}, 7 \mathrm{H}), 6.43(\mathrm{~d}, \mathrm{~J}=7.6 \mathrm{~Hz}, 1 \mathrm{H}), 5.36(\mathrm{~s}, 2 \mathrm{H}), 4.99-4.90(\mathrm{~m}, 1 \mathrm{H})$, $4.33(\mathrm{~d}, \mathrm{~J}=4.8 \mathrm{~Hz}, 1 \mathrm{H}), 4.21(\mathrm{~s}, 1 \mathrm{H}), 4.14(\mathrm{q}, \mathrm{J}=7.2 \mathrm{~Hz}, 2 \mathrm{H}), 4.06(\mathrm{~d}, \mathrm{~J}=5.6 \mathrm{~Hz}, 1 \mathrm{H})$, $3.86(\mathrm{~d}, \mathrm{~J}=11.6 \mathrm{~Hz}, 1 \mathrm{H}), 3.65(\mathrm{~d}, \mathrm{~J}=11.6 \mathrm{~Hz}, 1 \mathrm{H}), 3.32(\mathrm{~s}, 3 \mathrm{H}), 1.22(\mathrm{t}, \mathrm{J}=7.2 \mathrm{~Hz}, 3 \mathrm{H})$. ${ }^{13} \mathrm{C} \mathrm{NMR}\left(\mathrm{CDCl}_{3}\right) \delta 171.38,170.15,167.75,166.33,154.75,151.45,148.67,147.89$, $139.15,136.37,134.73,132.74,131.47,130.78,129.37,128.90,128.42,128.34,118.55$, $88.29,88.68,73.18,72.85,68.16,63.63,61.73,60.60,52.75,42.01,25.80,21.24,14.39$.

${ }^{31} \mathrm{P}$ NMR $\left(\mathrm{CDCl}_{3}\right) \delta$ 19.8. HRMS (MALDI): calcd for $\mathrm{C}_{35} \mathrm{H}_{36} \mathrm{~N}_{7} \mathrm{O}_{9} \mathrm{P}[\mathrm{M}+\mathrm{H}]=730.2390$, measured 730.1780. [High resolution NMR experimental data follows]

\section{Cumulative High Resolution NMR data for $9 \mathrm{~b}$.}

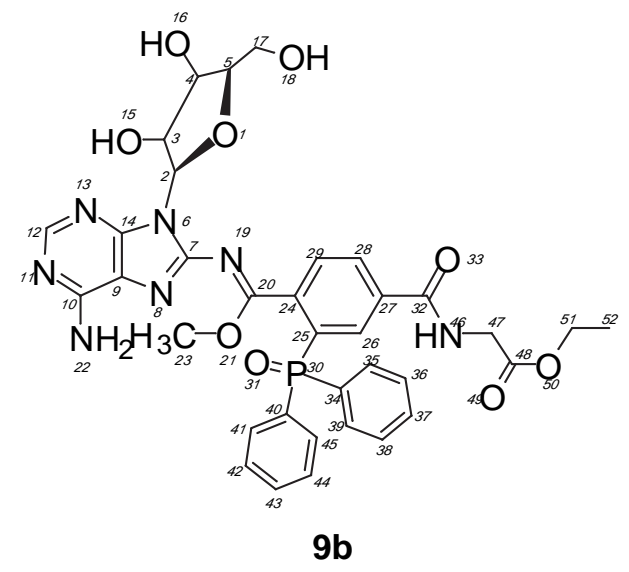

\section{${ }^{1} \mathrm{H}$ NMR (CDCl 3 , TMS Ref., $\left.500 \mathrm{MHz}\right)$ :}

$\delta 8.30\left(\mathrm{~d},{ }^{3} \mathrm{~J}\left({ }^{1} \mathrm{H},{ }^{31} \mathrm{P}\right)=14.2 \mathrm{~Hz}, 1 \mathrm{H}, 26\right)$

$\delta 8.07\left(\mathrm{~d},{ }^{3} J(28,29)=8.0 \mathrm{~Hz}, 1 \mathrm{H}, 28\right)$

$\delta 7.89\left(\mathrm{~d},{ }^{3} J(28,29)=8.0 \mathrm{~Hz}, 1 \mathrm{H}, 29\right)$

$\delta$ 7.78-7.88 (mm, 4H, 35,39,41,45)

$\delta 7.82(\mathrm{~s}, 1 \mathrm{H}, 12)$

$\delta 7.73\left(\mathrm{t},{ }^{3} J(46,47)=5.0 \mathrm{~Hz}, 1 \mathrm{H}, 46\right)$

$\delta 7.54(\mathrm{~m}, 2 \mathrm{H}, 37,43)$

$\delta 7.45(\mathrm{~m}, 4 \mathrm{H}, 36,38,42,44)$

$\delta 7.30$ (very broad)

$\delta 6.44\left(\mathrm{~d},{ }^{3} J(2,3)=7.4 \mathrm{~Hz}, 1 \mathrm{H}, 2\right)$

$\delta 5.17(\mathrm{~s}, 2 \mathrm{H}, 22)$

$\delta 4.98\left(\mathrm{dd}_{3}^{3} J(2,3)=7.4 \mathrm{~Hz},{ }^{3} J(3,4)=5.0 \mathrm{~Hz}, 1 \mathrm{H}, 3\right)$

$\delta 4.37\left(\mathrm{~d},{ }^{3} J(4,3)=4.9 \mathrm{~Hz}, 1 \mathrm{H}, 4\right)$

$\delta 4.24$ (s (broad), 1H, 5)

$\delta 4.18\left(\mathrm{q},{ }^{3} J(51,52)=7.1 \mathrm{~Hz}, 2 \mathrm{H}, 51\right)$

$\delta 4.10\left(\mathrm{~d},{ }^{3} J(46,47)=5.4 \mathrm{~Hz}, 2 \mathrm{H}, 47\right)$

$\delta 3.90\left(\mathrm{~d},{ }^{2} J\left(17,17^{\prime}\right)=12.5 \mathrm{~Hz}, 1 \mathrm{H}, 1^{\prime}\right)$

$\delta 3.69\left(\mathrm{~d},{ }^{2} J(17,17)=12.5 \mathrm{~Hz}, 1 \mathrm{H}, 17\right)$

$\delta 3.63$ (m (broad, distorted), $1 \mathrm{H}$, unassigned)

$\delta 3.33(\mathrm{~s}, 3 \mathrm{H}, 23)$

$\delta 1.25\left(\mathrm{t},{ }^{3} J(51,52)=7.2 \mathrm{~Hz}, 3 \mathrm{H}, 52\right)$

Notes: The last number(s) within the parentheses gives the assignment according to the numbering scheme used in the molecular drawing. Assignments were made from ${ }^{1} \mathrm{H} 1 \mathrm{D}$, gDQF-COSY and ROESY data. 


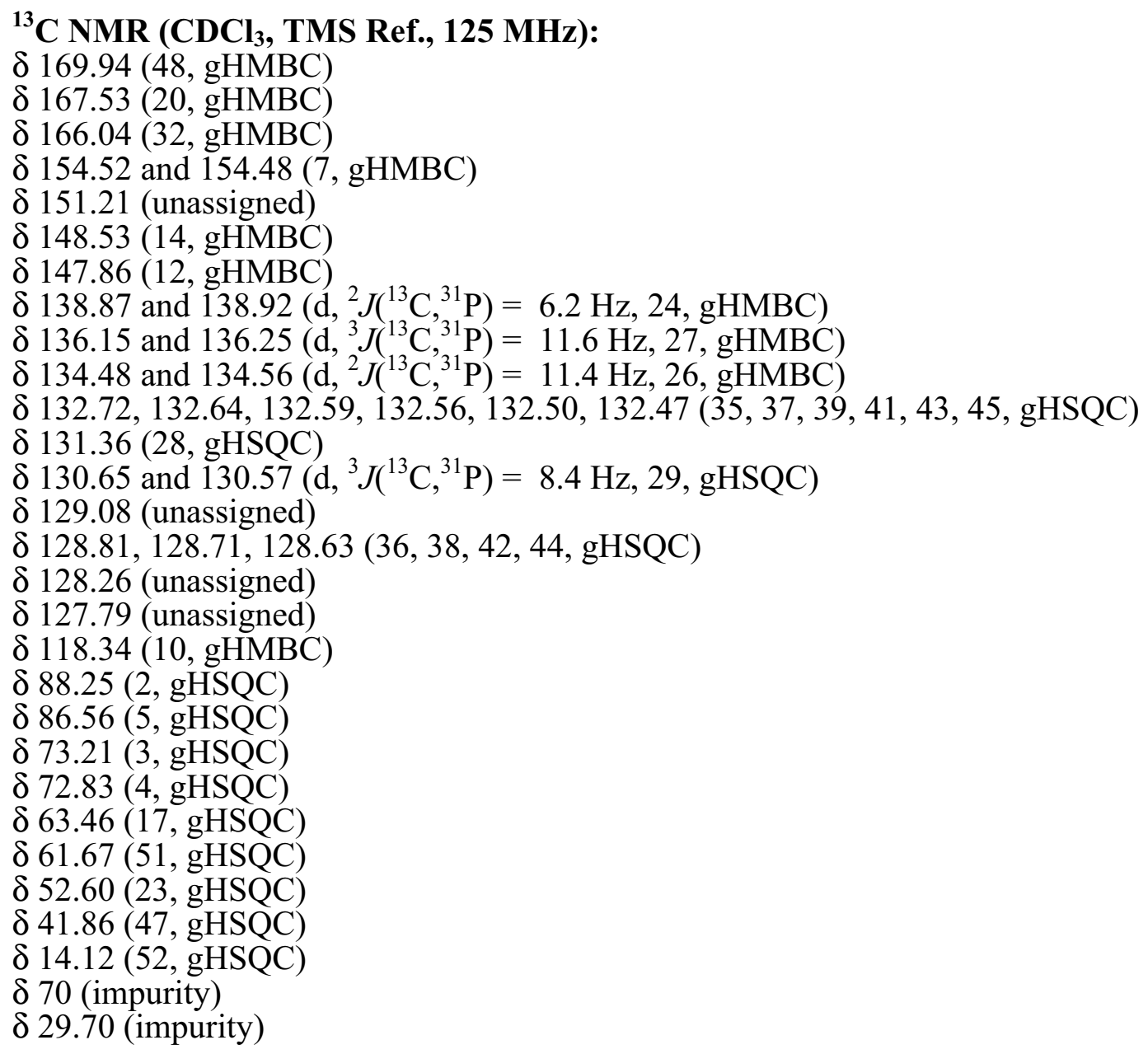

Notes: The last number within the parentheses gives the assignment according to the numbering scheme used in the molecular drawing. A subsequent experiment code indicates the experiment that was used to make the assignment; gHSQC: gradientselected hetero-nuclear single-quantum correlation, gHMBC: gradient-selected heteronuclear multiple-bond correlation.

\section{General procedure for preparation of esters:}

Note: 2-(diphenylphosphino) benzoic acid is available from Aldrich Chemical Corp. (cat. No. 45,488-5) and 2-diphenylphosphino-1-naphthoic acid was obtained from Alfa Aesar (cat. No. B22361).

To $\mathrm{PPh}_{3}(0.4898 \mathrm{mmol})$ in $2.3 \mathrm{~mL}$ anhydrous THF at $0^{\circ} \mathrm{C}$ was added DIAD $(0.4898$ $\mathrm{mmol})$. The reaction was stirred for $15 \mathrm{~min}$., followed by the addition of alcohol $(0.3265$ $\mathrm{mmol}$ ) in $1 \mathrm{~mL}$ anhydrous THF. After stirring for an additional $5 \mathrm{~min}$., the carboxylic acid $(0.3265 \mathrm{mmol})$ was added. The slurry was stirred for an additional $5 \mathrm{~min}$. and warmed to room temperature - the mixture was stirred until the disappearance of the precipitate. The solvent was removed in vacuo and resulting material chromatographed over silica [9:1 Pet Ether/ (4:2:1 EtOAc/ $\left.\left.\mathrm{CH}_{2} \mathrm{Cl}_{2} / \mathrm{MeOH}\right)\right]$ to yield the desired ester. 


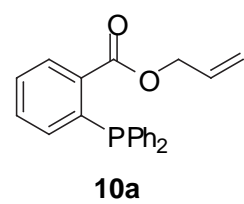

2-Diphenylphosphanyl-benzoic acid allyl ester (10a):

Reaction was carried out with allyl alcohol; yield $(0.099 \mathrm{~g}, 87.5 \%) .{ }^{1} \mathrm{H} \mathrm{NMR}\left(\mathrm{CDCl}_{3}\right) \delta$ 8.09-8.06 (m, 1H), 7.37-7.24 (m, 12H), 6.99-6.96 (m, 1H), 5.84 (ddt, J=17.2, 10.4, 5.6 $\mathrm{Hz}, 1 \mathrm{H}), 5.32(\mathrm{dd}, \mathrm{J}=17.2,1.2 \mathrm{~Hz}, 1 \mathrm{H}), 5.22(\mathrm{dd}, \mathrm{J}=10.4,1.2 \mathrm{~Hz}, 1 \mathrm{H}), 4.64$ (d, J= 5.6 $\mathrm{Hz}, 1 \mathrm{H}) ;{ }^{31} \mathrm{P}$ NMR $\left(\mathrm{CDCl}_{3}\right) \delta-3.35 ;{ }^{13} \mathrm{C} \mathrm{NMR}\left(\mathrm{CDCl}_{3}\right) \delta 166.5,140.8,140.5,138.1$, $138.0,134.5,134.4,134.3,134.2,133.9,132.1,130.8,130.8,128.7,128.6,128.5,128.3$, 118.5, 65.9. HRMALDI: calcd for $\mathrm{C}_{22} \mathrm{H}_{19} \mathrm{O}_{2} \mathrm{P}\left(\mathrm{M}+\mathrm{H}^{+}\right)$347.11, obsd 347.123.

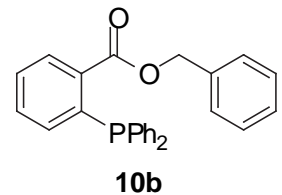

2-Diphenylphosphanyl-benzoic acid benzyl ester (10b):

Reaction was carried out with benzyl alcohol; yield $(0.116 \mathrm{~g}, 89.6 \%) .{ }^{1} \mathrm{H} \mathrm{NMR}\left(\mathrm{CDCl}_{3}\right)$ $\delta$ 8.09-8.06 (m, 1H), 7.37-7.24 (m, 17H), 6.95-6.92 (m, 1H), 5.19 (s, 2H); ${ }^{31} \mathrm{P}$ NMR $\left(\mathrm{CDCl}_{3}\right) \delta-3.44 ;{ }^{13} \mathrm{C} \mathrm{NMR}\left(\mathrm{CDCl}_{3}\right) \delta 166.7,140.9,140.7,138.4,138.1,138.0,135.9$, $135.3,134.5,134.4,134.2,134.2$, 134.0, 132.2, 131.0, 130.9, 128.8, 128.7, 128.6, 128.6, 128.5, 128.4, 128.3, 67.0. HRMALDI: calcd for $\mathrm{C}_{26} \mathrm{H}_{21} \mathrm{O}_{2} \mathrm{P}\left(\mathrm{M}+\mathrm{H}^{+}\right)$397.13, obsd 397.140 .

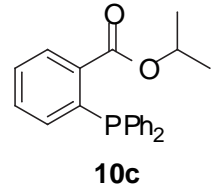

2-Diphenylphosphanyl-benzoic acid isopropyl ester (10c):

Reaction was carried out with isopropyl alcohol; yield $(0.105 \mathrm{~g}, 92.1 \%)$. ${ }^{1} \mathrm{H}$ NMR $\left(\mathrm{CDCl}_{3}\right) \delta$ 8.08-8.06 (m, 1H), 7.37-7.24 (m, 12H), 6.95-6.92 (m, 1H), 5.11 (sept, J=6.4 $\mathrm{Hz}, 1 \mathrm{H}), 1.17(\mathrm{~s}, 6 \mathrm{H}) ;{ }^{31} \mathrm{P} \mathrm{NMR}\left(\mathrm{CDCl}_{3}\right) \delta-3.69 ;{ }^{13} \mathrm{C} \mathrm{NMR}\left(\mathrm{CDCl}_{3}\right) \delta 166.6,140.2$, 139.9, 138.4, 138.3, 135.4, 135.3, 134.4, 134.2, 133.9, 133.8, 131.8, 130.7, 130.6, 128.9, 128.7, 128.6, 128.5, 128.3, 69.1, 21,9. HRMALDI: calcd for $\mathrm{C}_{22} \mathrm{H}_{21} \mathrm{O}_{2} \mathrm{P}\left(\mathrm{M}+\mathrm{H}^{+}\right)$ 349.13 , obsd 349.139 .

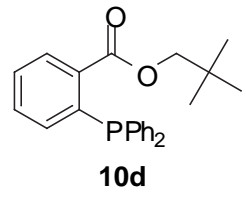

2-Diphenylphosphanyl-benzoic acid 2,2-dimethyl-propyl ester (10d):

Reaction was carried out with neopentyl alcohol; yield (0.102 g, 83.0\%). ${ }^{1} \mathrm{H}$ NMR $\left(\mathrm{CDCl}_{3}\right) \delta 8.10-8.07(\mathrm{~m}, 1 \mathrm{H}), 7.40-7.25(\mathrm{~m}, 12 \mathrm{H}), 6.96-6.93(\mathrm{~m}, 1 \mathrm{H}), 3.89(\mathrm{~s}, 2 \mathrm{H}), 0.94$ $(\mathrm{s}, 9 \mathrm{H}) ;{ }^{31} \mathrm{P}$ NMR $\left(\mathrm{CDCl}_{3}\right) \delta-3.73 ;{ }^{13} \mathrm{C} \mathrm{NMR}\left(\mathrm{CDCl}_{3}\right) \delta 166.8,141.0,140.8,138.2$, $138.1,134.7,134.5,134.1,133.9,133.8,132.0,130.5,130.5,128.8,128.7,128.6,128.6$, 128.5, 128.3 , 74.6, 31.6, 26.7. HRMALDI: calcd for $\mathrm{C}_{24} \mathrm{H}_{25} \mathrm{O}_{2} \mathrm{P}\left(\mathrm{M}+\mathrm{H}^{+}\right)$377.16, obsd 377.170 . 


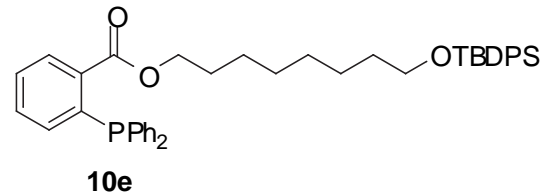

2-Diphenylphosphanyl-benzoic acid 8-(tert-butyldiphenylsilanyloxy)-octyl ester (10e):

Reaction was carried out with 8-(tert-Butyl-diphenyl-silanyloxy)-octan-1-ol; yield (0.169 g, $76.7 \%$ \%. ${ }^{1} \mathrm{H}$ NMR $\left(\mathrm{CDCl}_{3}\right) \delta 8.06-8.03(\mathrm{~m}, 1 \mathrm{H}), 7.69-7.66(\mathrm{~m}, 4 \mathrm{H}), 7.42-7.24(\mathrm{~m}$, $18 \mathrm{H}), 6.94-6.90(\mathrm{~m}, 1 \mathrm{H}), 4.14(\mathrm{t}, \mathrm{J}=6.8 \mathrm{~Hz}, 2 \mathrm{H}), 3.65(\mathrm{t}, \mathrm{J}=6.8 \mathrm{~Hz}, 2 \mathrm{H}), 1.58-1.52(\mathrm{~m}$, $4 \mathrm{H}), 1.34-1.24(\mathrm{~m}, 8 \mathrm{H}), 1.05(\mathrm{~s}, 9 \mathrm{H}) ;{ }^{31} \mathrm{P} \mathrm{NMR}\left(\mathrm{CDCl}_{3}\right) \delta-3.63 ;{ }^{13} \mathrm{C} \mathrm{NMR}\left(\mathrm{CDCl}_{3}\right) \delta$ $167.2,140.5,140.2,138.3,138.2,135.7,135.4,135.1,134.9,134.5,134.4,134.3,134.2$, 134.0, 133.9, 133.8, 132.1, 132.0, 130.7, 129.7, 128.8, 128.7, 128.7, 128.6, 128.6, 128.6, 128.4, 127.8, 65.6, 64.1, 32.7, 29.4, 28.6, 27.1, 26.1, 25.9, 19.4. HRMALDI: calcd for $\mathrm{C}_{43} \mathrm{H}_{49} \mathrm{O}_{3} \mathrm{PSi}\left(\mathrm{M}+\mathrm{H}^{+}\right)$673.32, obsd 673.336.

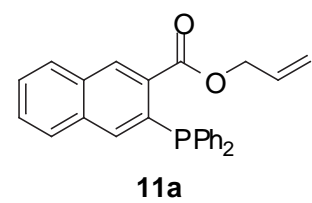

3-Diphenylphosphanyl-naphthalene-2-carboxylic acid allyl ester (11a)

${ }^{1} \mathrm{H} \mathrm{NMR}\left(\mathrm{CDCl}_{3}\right) \delta$ 7.93-7.91 $(\mathrm{m}, 1 \mathrm{H}), 7.77-7.772(\mathrm{~m}, 2 \mathrm{H}), 7.51-7.47(\mathrm{~m}, 2 \mathrm{H}), 7.32-7.26$ $(\mathrm{m}, 10 \mathrm{H}), 7.15(\mathrm{dd}, \mathrm{J}=8.4,3.2,1 \mathrm{H}), 5.84(\mathrm{ddt}, \mathrm{J}=17.0,10.4,6.0 \mathrm{~Hz}, 1 \mathrm{H}), 5.26(\mathrm{dd}, \mathrm{J}=$ 2.8., $1.6 \mathrm{~Hz}, 1 \mathrm{H}), 5.22(\mathrm{dd}, \mathrm{J}=2.8,1.6 \mathrm{~Hz}, 1 \mathrm{H}), 4.82(\mathrm{dt}, \mathrm{J}=6.0,1.2 \mathrm{~Hz}, 2 \mathrm{H}) ;{ }^{31} \mathrm{P} \mathrm{NMR}$ $\left(\mathrm{CDCl}_{3}\right) \delta-8.14 ;{ }^{13} \mathrm{C} \mathrm{NMR}\left(\mathrm{CDCl}_{3}\right) \delta 169.0,139.2,138.9,137.1,136.9,134.1,133.9$, $133.7,133.6,133.4,133.2,131.9,130.1,130.0,129.8,129.0,128.9,128.8,128.7,128.4$, 127.7, 127.5, 125.5, 119.2, 70.2. HRMALDI: calcd for $\mathrm{C}_{26} \mathrm{H}_{21} \mathrm{O}_{2} \mathrm{P}\left(\mathrm{M}+\mathrm{H}^{+}\right)$: 397.135, measured: 397.120 .

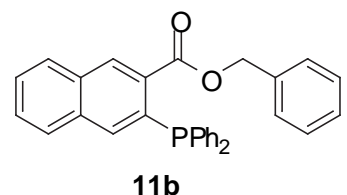

3-Diphenylphosphanyl-naphthalene-2-carboxylic acid benzyl ester (11b)

${ }^{1} \mathrm{H}$ NMR $\left(\mathrm{CDCl}_{3}\right) \delta$ 7.90-7.85 (m, 1H), 7.80-7.72 (m, 2H), 7.52-7.47 (m, 2H), 7.32-7.26 $(\mathrm{m}, 10 \mathrm{H}), 7.21-7.25(\mathrm{~m}, 5 \mathrm{H}), 7.20(\mathrm{dd}, \mathrm{J}=8.4,3.2,1 \mathrm{H}), 5.40(\mathrm{~s}, 2 \mathrm{H}) ;{ }^{31} \mathrm{P}$ NMR $\left(\mathrm{CDCl}_{3}\right) \delta$ -8.31; ${ }^{13} \mathrm{C}$ NMR $\left(\mathrm{CDCl}_{3}\right) \delta 169.3,139.5,139.2,137.1,137.0,135.5,134.1,133.9,133.7$, 133.6, 133.2, 133.0, 130.1, 130.0, 129.9, 129.0, 128.9, 128.4, 127.7, 127.6, 125.5, 70.3. HRMALDI: calcd for $\mathrm{C}_{26} \mathrm{H}_{21} \mathrm{O}_{2} \mathrm{P}\left(\mathrm{M}+\mathrm{H}^{+}\right)$: 447.151, measured: 447.148. 


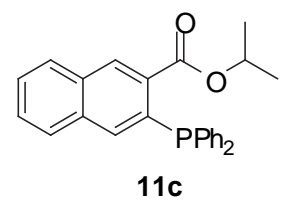

3-Diphenylphosphanyl-naphthalene-2-carboxylic acid isopropyl ester (11c) ${ }^{1} \mathrm{H} \mathrm{NMR}\left(\mathrm{CDCl}_{3}\right) \delta 8.00(\mathrm{dd}, \mathrm{J}=8.8,1.2 \mathrm{~Hz}, 1 \mathrm{H}), 7.85-7.80(\mathrm{~m}, 2 \mathrm{H}), 7.62-7.54(\mathrm{~m}, 2 \mathrm{H})$, 7.35-7.38 (m, 10H), $7.20(\mathrm{dd}, \mathrm{J}=8.4,3.2,1 \mathrm{H}), 5.45(\mathrm{sept}, \mathrm{J}=6 \mathrm{~Hz}, 1 \mathrm{H}), 1.33(\mathrm{~d}, \mathrm{~J}=6.4 \mathrm{~Hz}$, $6 \mathrm{H}) ;{ }^{31} \mathrm{P} \mathrm{NMR}\left(\mathrm{CDCl}_{3}\right) \delta-9.39 ;{ }^{13} \mathrm{C}$ NMR $\left(\mathrm{CDCl}_{3}\right) \delta 168.9,137.2,137.1,134.1,133.9$, 133.7, 133.6, 132.4, 132.2, 130.0, 129.9, 129.8, 128.8, 128.7, 128.4, 127.6, 127.4, 125.5, 70.01, 21.9. HRMALDI: calcd for $\mathrm{C}_{26} \mathrm{H}_{23} \mathrm{O}_{2} \mathrm{P}\left(\mathrm{M}+\mathrm{H}^{+}\right)$: 399.151, measured: 399.145

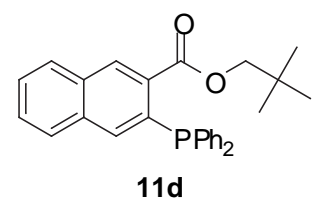

3-Diphenylphosphanyl-naphthalene-2-carboxylic acid 2,2-dimethyl-propyl ester (11d)

${ }^{1} \mathrm{H} \mathrm{NMR}\left(\mathrm{CDCl}_{3}\right) \delta$ 7.98-7.90 (m, 1H), 7.83-776(m, 2H), 7.57-7.49 (m, 2H), 7.37-7.29 $(\mathrm{m}, 10 \mathrm{H}), 7.23-7.15(\mathrm{~m}, 1 \mathrm{H}), 4.18(\mathrm{~s}, 2 \mathrm{H}) 0.90(\mathrm{~s}, 9 \mathrm{H}) ;{ }^{31} \mathrm{P} \mathrm{NMR}\left(\mathrm{CDCl}_{3}\right) \delta-8.71 ;{ }^{13} \mathrm{C}$ $\operatorname{NMR}\left(\mathrm{CDCl}_{3}\right) \delta 169.8,137.9,137.1,137.0,133.9,133.8,133.7,133.6,132.7,132.5$, $130.1,129.9,129.8,129.0,128.8,128.7,128.3,127.9,127.6,127.5,125.6,75.1,31.4$, 26.7. HRMALDI: calcd for $\mathrm{C}_{28} \mathrm{H}_{27} \mathrm{O}_{2} \mathrm{P}\left(\mathrm{M}+\mathrm{H}^{+}\right)$: 427.182 , measured: 427.165

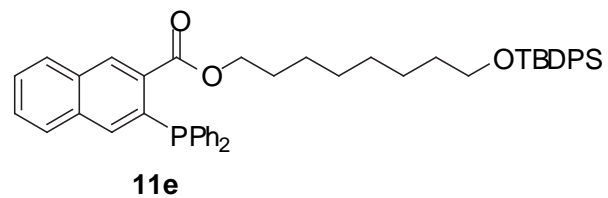

3-Diphenylphosphanyl-naphthalene-2-carboxylic acid 8-(tert-butryl-diphenylsilanyloxy)-octyl ester (11e)

${ }^{1} \mathrm{H}$ NMR $\left(\mathrm{CDCl}_{3}\right) \delta$ 7.97-7.94 (m, 1H), 7.80-7.34 (m, 2H), 7.68-765 (m, 4H), 7.55-7.48 $(\mathrm{m}, 2 \mathrm{H}), 7.39-7.28(\mathrm{~m}, 16 \mathrm{H}), 7.18-7.15(\mathrm{~m}, 1 \mathrm{H}), 4.36(\mathrm{t}, \mathrm{J}=6.4 \mathrm{~Hz}, 2 \mathrm{H}), 3.64(\mathrm{t}, \mathrm{J}=6.4$ $\mathrm{Hz}, 2 \mathrm{H}), 1.57-1.49(\mathrm{~m}, 4 \mathrm{H}), 1.32-1.18(\mathrm{~m}, 8 \mathrm{H}), 1.04(\mathrm{~s}, 9 \mathrm{H}) ;{ }^{31} \mathrm{P} \mathrm{NMR}\left(\mathrm{CDCl}_{3}\right) \delta-8.35$;

${ }^{13} \mathrm{C} \mathrm{NMR}\left(\mathrm{CDCl}_{3}\right) \delta 169.5,142.2,140.0,139.6,137.3,137.2,135.9,134.4,133.8,133.7$, 133.6, 133.0, 132.9, 130.1, 130.0, 129.9, 129.8, 129.0, 128.9, 128.8, 128.7, 128.4, 127.9, 127.7, 127.5, 125.6, 66.0, 64.2, 32.8, 29.4, 28.7, 27.2, 26.2, 19.5, 14.5. HRMALDI: calcd for $\mathrm{C}_{47} \mathrm{H}_{51} \mathrm{O}_{4} \mathrm{PSi}\left(\mathrm{M}+\mathrm{H}^{+}\right)$: 739.337, measured: 739.330 .

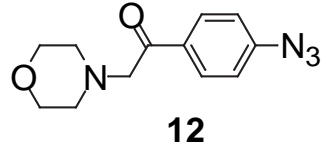

1-(4-Azido-phenyl)-2-morpholin-4-yl-ethanone (12).

To $37.7 \mathrm{mg}$ of tetrahydro-1,4-oxazine $(0.43 \mathrm{mmol})$ was added $2 \mathrm{~mL}$ anhydrous THF along with $62.4 \mu \mathrm{L}$ distilled triethylamine $(0.45 \mathrm{mmol})$. The solution was chilled to $0^{\circ} \mathrm{C}$ for 5 min followed by the addition of $51.8 \mathrm{mg} 4$-azidophenacyl bromide $(0.22 \mathrm{mmol})$. The 
solution was stirred and sheltered from light at $0^{\circ} \mathrm{C}$ for $2 \mathrm{~h}$ after which time TLC (7:3:2:0.5 Hex:EtOAc: $\mathrm{CH}_{2} \mathrm{Cl}_{2}: \mathrm{MeOH}$ ) revealed complete consumption of the 4azidophenacyl bromide and generation of a new $\mathrm{UV}$-active spot with $\mathrm{R}_{\mathrm{f}} \sim 0.25$. The solid triethylamine hydrochloride was filtered from the solution and the THF removed in vacuo. PTLC using the TLC conditions noted above afforded $46 \mathrm{mg}$ of aryl azide 12 in $85 \%$ yield. ${ }^{1} \mathrm{H}$ NMR $\left(\mathrm{CDCl}_{3}\right) \delta 8.05-8.04(\mathrm{~m}, 1 \mathrm{H}), 8.03-8.02(\mathrm{~m}, 1 \mathrm{H}), 7.10-7.09(\mathrm{~m}$, $1 \mathrm{H})$, 7.08-7.07 (m, 1H), 3.78-3.75 (m, 6H), 2.61-2.59 (m, 4H). ${ }^{13} \mathrm{C} \mathrm{NMR}\left(\mathrm{CDCl}_{3}\right) \delta$ 194.85, 145.35, 132.75, 130.40, 119.17, 66.97, 64.94, 54.03. LRMS (ESI) calcd for $\mathrm{C}_{12} \mathrm{H}_{14} \mathrm{~N}_{4} \mathrm{O}_{2}[\mathrm{M}+\mathrm{H}]=247.12$, measured 247.0. HR mass spectral data not attainable due to azide lability.

1-Azido-4-butyl-benzene (13)

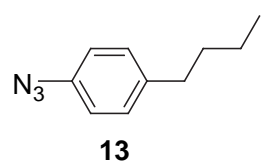

${ }^{1} \mathrm{H} \mathrm{NMR}\left(\mathrm{CDCl}_{3}\right) \delta 7.19(\mathrm{~d}, \mathrm{~J}=8.4 \mathrm{~Hz}, 2 \mathrm{H}), 7.97(\mathrm{~d}, \mathrm{~J}=8.4 \mathrm{~Hz}, 2 \mathrm{H}), 2.62(\mathrm{t}, \mathrm{J}=7.6 \mathrm{~Hz}$, $2 \mathrm{H}), 1.67-1.57$ (m, 2H), 1.412-1.35 (m, 2H), $0.965(\mathrm{t}, \mathrm{J}=7.6 \mathrm{~Hz}, 3 \mathrm{H}) ;{ }^{13} \mathrm{C} \mathrm{NMR}\left(\mathrm{CDCl}_{3}\right)$ $\delta$ 140.0, 137.5, 130.0, 119.1, 35.2, 33.9, 22.5, 14.2. ESI: calcd for $\mathrm{C}_{10} \mathrm{H}_{13} \mathrm{~N}_{3}\left(\mathrm{M}+\mathrm{H}^{+}\right)$: 176.11, measured: 176.10. HR mass spectral data not attainable due to azide lability

General Procedure for Staudinger Ligations (for phosphines 10a-e, 11a-e):

To the aryl azide $(0.0124 \mathrm{~g}, 0.0505 \mathrm{mmol})$ in $252.5 \mu \mathrm{L} \mathrm{1:1} \mathrm{THF} / \mathrm{H}_{2} \mathrm{O}$ was added the phosphine $(0.0200 \mathrm{~g}, 0.0505 \mathrm{mmol})$ in $252.5 \mu \mathrm{L} 1: 1 \mathrm{THF} / \mathrm{H}_{2} \mathrm{O}$. The reaction was incubated at RT for $1.5 \mathrm{~h}$. The product was extracted into EtOAc, washed with brine, and dried over $\mathrm{Na}_{2} \mathrm{SO}_{4}$. The solvent was removed in vacuo and the resulting material was chromatographed [9:1 Pet Ether/ $\left(4: 2: 1 \mathrm{EtOAc} / \mathrm{CH}_{2} \mathrm{Cl}_{2} / \mathrm{MeOH}\right]$ to yield the desired conjugate as a light yellow oil.

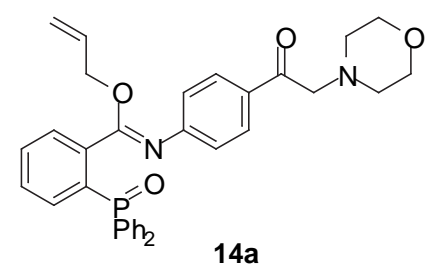

2-(Diphenyl-phosphinoyl)-N-[4-(2-morpholin-4-yl-acetyl)-phenyl]-benzimidic acid allyl ester (14a) :

Reaction was carried out with 2-Diphenylphosphanyl-benzoic acid allyl ester and 1-(4Azido-phenyl)-2-morpholin-4-yl-ethanone; yield $(0.0210 \mathrm{~g}, 64.4 \%) .{ }^{1} \mathrm{H} \mathrm{NMR}\left(\mathrm{CDCl}_{3}\right) \delta$ 7.88-7.85 (m, 1H), 7.70-7.61 (m, 7H), 7.57-7.52 (m, 4H), $7.47(\mathrm{td}, \mathrm{J}=7.6,2.8 \mathrm{~Hz}, 4 \mathrm{H})$, $6.63(\mathrm{~d}, \mathrm{~J}=8.8 \mathrm{~Hz}, 2 \mathrm{H}), 5.51(\mathrm{ddt}, \mathrm{J}=17.2,10.4,6.4 \mathrm{~Hz}, 1 \mathrm{H}), 5.04(\mathrm{dd}, \mathrm{J}=17.6,1.2 \mathrm{~Hz}$, $1 \mathrm{H}), 5.02(\mathrm{dd}, \mathrm{J}=10.4,1.2 \mathrm{~Hz}, 1 \mathrm{H}), 4.35(\mathrm{~d}, \mathrm{~J}=6.0 \mathrm{~Hz}, 2 \mathrm{H}), 3.77-3.75(\mathrm{~m}, 4 \mathrm{H}), 3.69(\mathrm{~s}$, $2 \mathrm{H}), 2.57-2.54(\mathrm{~m}, 4 \mathrm{H}) ;{ }^{31} \mathrm{P}$ NMR $\left(\mathrm{CDCl}_{3}\right) \delta 9.13 ;{ }^{13} \mathrm{C} \mathrm{NMR}\left(\mathrm{CDCl}_{3}\right) \delta 194.2,167.4$, 137.1, 137.1, 134.7, 134.6, 132.5, 132.4, 132.3, 132.2, 132.1, 132.1, 131.6, 131.3, 131.1, 131.0, 130.7, 130.7, 138.4, 130.3, 129.8, 129.0, 128.9, 125.4, 122.6, 122.4, 118.7, 67.1, 66.5, 64.2, 54.2. HRMALDI: calcd for $\mathrm{C}_{34} \mathrm{H}_{33} \mathrm{~N}_{2} \mathrm{O}_{4} \mathrm{P}\left(\mathrm{M}+\mathrm{H}^{+}\right)$565.22, obsd 565.216. 


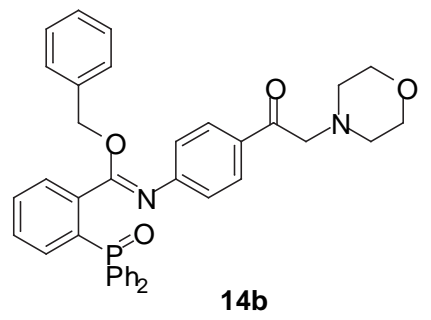

2-(Diphenyl-phosphinoyl)-N-[4-(2-morpholin-4-yl-acetyl)-phenyl]-benzimidic acid benzyl ester (14b):

Reaction was carried out with 2-Diphenylphosphanyl-benzoic acid benzyl ester and 1-(4Azido-phenyl)-2-morpholin-4-yl-ethanone; yield (0.0164 g, 52.9\%). ${ }^{1} \mathrm{H} \mathrm{NMR}\left(\mathrm{CDCl}_{3}\right) \delta$ $7.86(\mathrm{dd}, \mathrm{J}=7.2,3.6 \mathrm{~Hz}, 1 \mathrm{H}), 7.69-7.63(\mathrm{~m}, 7 \mathrm{H}), 7.54-7.51(\mathrm{~m}, 4 \mathrm{H}), 7.42(\mathrm{td}, \mathrm{J}=7.6,2.8$ $\mathrm{Hz}, 4 \mathrm{H}), 7.26-7.17(\mathrm{~m}, 3 \mathrm{H}), 6.98(\mathrm{~d}, \mathrm{~J}=8.8 \mathrm{~Hz}, 2 \mathrm{H}), 6.66(\mathrm{~d}, \mathrm{~J}=8.8 \mathrm{~Hz}, 2 \mathrm{H}), 4.91(\mathrm{~s}$, $2 \mathrm{H}), 3.77-3.75(\mathrm{~m}, 4 \mathrm{H}), 3.68(\mathrm{~s}, 2 \mathrm{H}), 2.57-2.54(\mathrm{~m}, 4 \mathrm{H}) ;{ }^{31} \mathrm{P} \mathrm{NMR}\left(\mathrm{CDCl}_{3}\right) \delta 9.05 ;{ }^{13} \mathrm{C}$ NMR $\left(\mathrm{CDCl}_{3}\right) \delta 194.2,167.6,137.1,137.1,135.3,134.7,134.6,132.5,132.4,132.3$, 132.2 , 132.1, 132.1, 131.2, 131,1, 130.9, 130.8, 130.7, 130.2, 129.8, 129.8, 128.9, 128.8, 128.5, 128.4, 128.2, 125.5, 122.6, 122.4, 67.4, 67.0, 64.2, 54.2. HRMALDI: calcd for $\mathrm{C}_{38} \mathrm{H}_{35} \mathrm{~N}_{2} \mathrm{O}_{4} \mathrm{P}\left(\mathrm{M}+\mathrm{H}^{+}\right)$615.23, obsd 615.231.

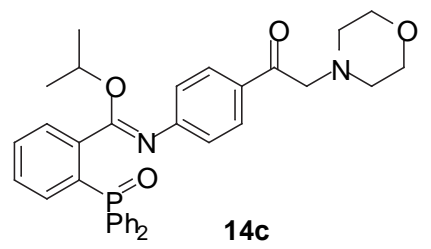

2-(Diphenyl-phosphinoyl)-N-[4-(2-morpholin-4-yl-acetyl)-phenyl]-benzimidic acid isopropyl ester (14c):

Reaction was carried out with 2-Diphenylphosphanyl-benzoic acid isopropyl ester and 1(4-Azido-phenyl)-2-morpholin-4-yl-ethanone; yield (0.0213 g, 65.5\%). ${ }^{1} \mathrm{H}$ NMR

$\left(\mathrm{CDCl}_{3}\right) \delta$ 7.90-7.85 (m, 1H), 7.71-7.61 (m, 8H), 7.56-7.52 (m, 3H), $7.46(\mathrm{td}, \mathrm{J}=7.6,3.2$ $\mathrm{Hz}, 4 \mathrm{H}), 6.62$ (d, J=8.8 Hz, 2H), 4.78 (sept, J=6.4 Hz, 1H), 3.77-3.75 (m, 4H), 3.68 (s, $2 \mathrm{H}), 2.57-2.54(\mathrm{~m}, 4 \mathrm{H}), 0.97(\mathrm{~d}, \mathrm{~J}=6.4 \mathrm{~Hz}, 6 \mathrm{H}) ;{ }^{31} \mathrm{P}$ NMR $\left(\mathrm{CDCl}_{3}\right) \delta 9.73 ;{ }^{13} \mathrm{C} \mathrm{NMR}$ $\left(\mathrm{CDCl}_{3}\right) \delta 194.2,166.7,137.3,137.3,135.2,135.1,132.6,132.5,132.3,132.0,132.0$, 131.2, 131.1, 131.0, 130.9, 129.8, 128.9, 128.8, 125.4, 122.6, 122.4, 67.1, 66.1, 64.2, 54.2, 21.5. HRMALDI: calcd for $\mathrm{C}_{34} \mathrm{H}_{35} \mathrm{~N}_{2} \mathrm{O}_{4} \mathrm{P}\left(\mathrm{M}+\mathrm{H}^{+}\right)$567.23, obsd 567.228.

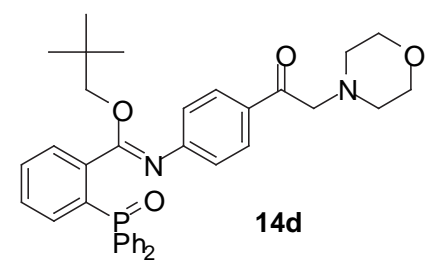

2-(Diphenyl-phosphinoyl)-N-[4-(2-morpholin-4-yl-acetyl)-phenyl]-benzimidic acid (2,2-dimethyl-propyl) ester (14d):

Reaction was carried out with 2-Diphenylphosphanyl-benzoic acid 2,2-dimethyl-propyl ester and 1-(4-Azido-phenyl)-2-morpholin-4-yl-ethanone; yield $(0.0211 \mathrm{~g}, 66.8 \%)$. ${ }^{1} \mathrm{H}$ $\operatorname{NMR}\left(\mathrm{CDCl}_{3}\right) \delta$ 7.90-7.85 (m, 1H), 7.71-7.61 (m, 8H), 7.59-7.52 (m, 3H), 7.47-7.43 (m, 
$4 \mathrm{H}), 6.63(\mathrm{~d}, \mathrm{~J}=8.8 \mathrm{~Hz}, 2 \mathrm{H}), 3.77-3.75(\mathrm{~m}, 4 \mathrm{H}), 3.68(\mathrm{~s}, 2 \mathrm{H}), 3.58(\mathrm{~s}, 2 \mathrm{H}), 2.57-2.254$ $(\mathrm{m}, 4 \mathrm{H}), 0.77(\mathrm{~s}, 9 \mathrm{H}) ;{ }^{31} \mathrm{P}$ NMR $\left(\mathrm{CDCl}_{3}\right) \delta 9.38 ;{ }^{13} \mathrm{C} \mathrm{NMR}\left(\mathrm{CDCl}_{3}\right) \delta 194.1,167.3,137.1$, 137.0, 135.2, 135.1, 132.5, 132.4, 132.3, 132.0, 132.0, 131.2, 131.1, 130.5, 130.4, 129.8, 129.7, 128.9, 128.8, 125.4, 122.6, 122.4, 74.8, 67.1, 64.2, 54.2, 31.4, 26.5. HRMALDI: calcd for $\mathrm{C}_{36} \mathrm{H}_{39} \mathrm{~N}_{2} \mathrm{O}_{4} \mathrm{P}\left(\mathrm{M}+\mathrm{H}^{+}\right)$595.26, obsd 595.244.

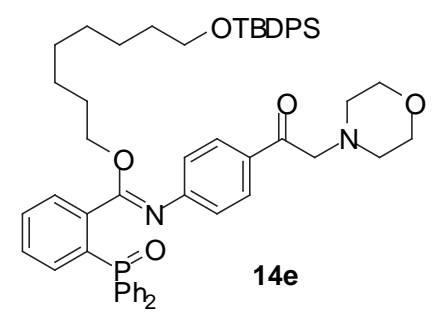

2-(Diphenyl-phosphinoyl)-N-[4-(2-morpholin-4-yl-acetyl)-phenyl]-benzimidic acid [8-(tert-Butyl-diphenyl-silanyloxy)-octyl] ester (14e):

Reaction was carried out with 2-Diphenylphosphanyl-benzoic acid 8-(tert-butyldiphenyl-silanyloxy)-octyl ester and 1-(4-Azido-phenyl)-2-morpholin-4-yl-ethanone; yield $(0.0347 \mathrm{~g}, 65.6 \%)$. ${ }^{1} \mathrm{H}$ NMR $\left(\mathrm{CDCl}_{3}\right) \delta$ 7.87-7.84 $(\mathrm{m}, 1 \mathrm{H}), 7.70-7.64(\mathrm{~m}, 11 \mathrm{H})$, 7.55-7.51 (m, 4H), 7.40-7.34 (m, 10H), $6.63(\mathrm{~d}, \mathrm{~J}=8.8 \mathrm{~Hz}, 2 \mathrm{H}), 3.82(\mathrm{t}, \mathrm{J}=6.8 \mathrm{~Hz}, 2 \mathrm{H})$, 3.77-3.75 (m, 4H), $3.68(\mathrm{~s}, 2 \mathrm{H}), 3.64(\mathrm{t}, \mathrm{J}=6.8 \mathrm{~Hz}, 2 \mathrm{H}), 2.57-2.52(\mathrm{~m}, 4 \mathrm{H}), 1.59-1.49(\mathrm{~m}$, $2 \mathrm{H}), 1.35-1.20(\mathrm{~m}, 4 \mathrm{H}), 1.17-1.09(\mathrm{~m}, 6 \mathrm{H}), 1.04(\mathrm{~s}, 9 \mathrm{H}) ;{ }^{31} \mathrm{P} \mathrm{NMR}\left(\mathrm{CDCl}_{3}\right) \delta 9.18 ;{ }^{13} \mathrm{C}$ NMR $\left(\mathrm{CDCl}_{3}\right) \delta 194.1,167.7,137.4,137.3,135.7,134.9,134.8,134.3,132.5,132.4$, 132.3, 132.2, 132.1, 132.0, 131.0, 130.9, 130.7, 130.7, 130.4, 129.8, 129.7, 129.7, 129.1, $128.9,128.8,128.5,128.4,127.7,125.4,122.5,122.4,119.1,67.0,67.0,66.0,64.1,54.2$, 32.7, 29.3, 29.3, 28.1, 27.0, 25.9, 25.8, 19.4. HRMALDI: calcd for $\mathrm{C}_{55} \mathrm{H}_{63} \mathrm{~N}_{2} \mathrm{O}_{5} \mathrm{PSi}(\mathrm{M}+$ $\left.\mathrm{H}^{+}\right)$891.42, obsd 891.331.

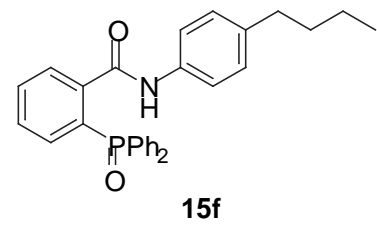

$\mathrm{N}$-(4-butylphenyl)-2-(diphenylphosphoryl)benzamide (15f):

${ }^{1} \mathrm{H}$ NMR $\left(\mathrm{CDCl}_{3}\right) \delta 10.54(\mathrm{bs}, 1 \mathrm{H}), 8.09-8.06(\mathrm{~m}, 1 \mathrm{H}), 7.70-7.63(\mathrm{~m}, 5 \mathrm{H}), 7.53-7.49(\mathrm{~m}$, $2 \mathrm{H})$, 7.44-7.39 (m, 5H), 7.32-7.30 (m, 2H), 7.15-7.09 (m, 1H), 7.01 (d, J= 8.4 Hz, 2H), $2.54(\mathrm{t}, \mathrm{J}=7.6 \mathrm{~Hz}, 2 \mathrm{H}), 1.59-1.52(\mathrm{~m}, 2 \mathrm{H}), 1.37-1.28(\mathrm{~m}, 2 \mathrm{H}), 0.92(\mathrm{t}, \mathrm{J}=7.6 \mathrm{~Hz}, 3 \mathrm{H}) ;{ }^{31} \mathrm{P}$ $\operatorname{NMR}\left(\mathrm{CDCl}_{3}\right) \delta 36.36 ;{ }^{13} \mathrm{C} \mathrm{NMR}\left(\mathrm{CDCl}_{3}\right) \delta 165.4,141.7,141.6,139.0,135.7,133.5$, $133,4,133.06,132.97,132.6,132.55,132.49,132.4,131.8,131.7,131.3,130.32,130.28$, $130.23,131.17,129.3,129.0,128.8,128.6,120.3,35.3,33.9,22.4,14.2$. HRMALDI: calcd for $\mathrm{C}_{29} \mathrm{H}_{28} \mathrm{NO}_{2} \mathrm{P}\left(\mathrm{M}+\mathrm{H}^{+}\right) 454.19$, obsd 454.181. 


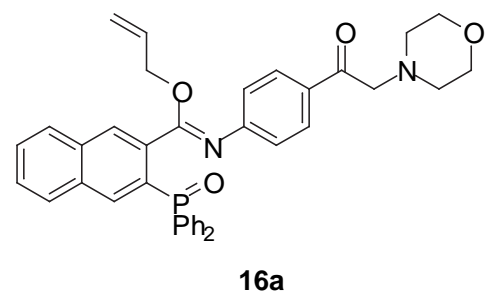

3-(Diphenyl-phosphinoyl)-N-[4-(2-morpholin-4-yl-acetyl)-phenyl]-naphthalene-2carboximidic acid allyl ester (16a).

${ }^{1} \mathrm{H}$ NMR $\left(\mathrm{CDCl}_{3}\right) \delta 9.17(\mathrm{~s}, 1 \mathrm{H}), 8.31(\mathrm{~d}, \mathrm{~J}=8 \mathrm{~Hz}, 1 \mathrm{H}), 7.87-784(\mathrm{~m}, 2 \mathrm{H}), 7.77-7.69(\mathrm{~m}$, 4H), 7.64-7.35 (m, 6H), $7.31(\mathrm{~d}, \mathrm{~J}=8.4 \mathrm{~Hz}, 2 \mathrm{H}), 7.24(\mathrm{dd}, \mathrm{J}=12.0,8.0 \mathrm{~Hz}, 2 \mathrm{H}), 7.05$ (d, $\mathrm{J}=8.4 \mathrm{~Hz}, 2 \mathrm{H}), 5.78(\mathrm{ddt}, \mathrm{J}=17.0,10.4,6.0 \mathrm{~Hz}, 1 \mathrm{H}), 5.21(\mathrm{dd}, \mathrm{J}=17.2,1.2 \mathrm{~Hz}, 1 \mathrm{H})$, $5.12(\mathrm{dd}, \mathrm{J}=10.4,1.2 \mathrm{~Hz}, 1 \mathrm{H}), 4.73(\mathrm{~d}, \mathrm{~J}=6.4 \mathrm{~Hz}, 2 \mathrm{H}), 3.77-3.75(\mathrm{~m}, 4 \mathrm{H}), 3.69(\mathrm{~s}, 2 \mathrm{H})$, 2.57-2.52 (m, 4H); ${ }^{31} \mathrm{P}$ NMR $\left(\mathrm{CDCl}_{3}\right) \delta 33.32 ;{ }^{13} \mathrm{C} \mathrm{NMR}\left(\mathrm{CDCl}_{3}\right) \delta 195.0,166.9,151.2$, 137.9, 137.5, 136.7, 135.0,134.2, 133.0, 131.8, 131.5, 130.4, 129.6, 128.7, 128.0, 127.1, 125.9, 121.8, 115.1, 67.1, 66.5, 64.2, 54.2. HRMALDI: calcd for $\mathrm{C}_{38} \mathrm{H}_{35} \mathrm{~N}_{2} \mathrm{O}_{4} \mathrm{P}(\mathrm{M}+$ $\mathrm{H}^{+}$): 615.241, measured: 615.225

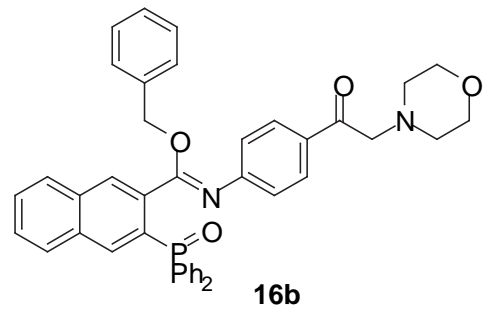

3-(Diphenyl-phophinoyl-Np[4-2-morpholin-4-yl-acetyl)-phenyl]-naphthalene-2carboximidic acid benzyl ester (16b)

${ }^{1} \mathrm{H}$ NMR $\left(\mathrm{CDCl}_{3}\right) \delta 9.20(\mathrm{~s}, 1 \mathrm{H}), 8.30(\mathrm{~d}, \mathrm{~J}=8 \mathrm{~Hz}, 1 \mathrm{H}), 7.87-783(\mathrm{~m}, 2 \mathrm{H}), 7.75-7.70(\mathrm{~m}$, 4H), 7.63-7.22 (m, 15H), $7.04(\mathrm{~d}, \mathrm{~J}=8.8 \mathrm{~Hz}, 2 \mathrm{H}), 4.65(\mathrm{~s}, 2 \mathrm{H}), 3.77-3.75(\mathrm{~m}, 4 \mathrm{H}), 3.68$ $(\mathrm{s}, 2 \mathrm{H}), 2.57-2.52(\mathrm{~m}, 4 \mathrm{H}) ;{ }^{31} \mathrm{P}$ NMR $\left(\mathrm{CDCl}_{3}\right) \delta 33.26 ;{ }^{13} \mathrm{C} \mathrm{NMR}\left(\mathrm{CDCl}_{3}\right) \delta 195.0,166.2$, $150.4,140.6,137.9,136.6,135.1,134.2,132.9$, 131.5, 130.4, 129.6, 128.7, 128.6, 128.0, 127.3, 127.1, 125.9, 121.8, 67.9, 67.0, 64.2, 54.2. HRMALDI: calcd for $\mathrm{C}_{42} \mathrm{H}_{37} \mathrm{~N}_{2} \mathrm{O}_{4} \mathrm{P}$ $\left(\mathrm{M}+\mathrm{H}^{+}\right): 665.256$, measured: 665.241

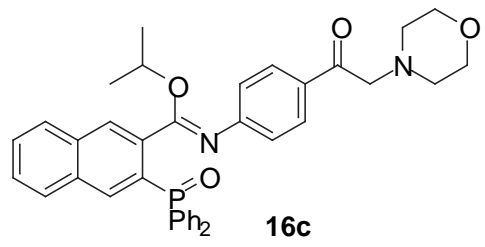

3-(Diphenyl-phosphinoyl)-N-[4-(2-morpholin-4-yl-acetyl)-phenyl]-naphthalene-2carboximidic acid isopropyl ester (16c)

${ }^{1} \mathrm{H} \mathrm{NMR}\left(\mathrm{CDCl}_{3}\right) \delta 9.20(\mathrm{~s}, 1 \mathrm{H}), 8.29(\mathrm{~d}, \mathrm{~J}=8 \mathrm{~Hz}, 1 \mathrm{H}), 7.86-784(\mathrm{~m}, 2 \mathrm{H}), 7.75-7.71(\mathrm{~m}$, $4 \mathrm{H}), 7.64-7.33(\mathrm{~m}, 6 \mathrm{H}), 7.30(\mathrm{~d}, \mathrm{~J}=8.4 \mathrm{~Hz}, 2 \mathrm{H}), 7.25(\mathrm{dd}, \mathrm{J}=12.0,8.0 \mathrm{~Hz}, 2 \mathrm{H}), 7.05(\mathrm{~d}$, $\mathrm{J}=8.4 \mathrm{~Hz}, 2 \mathrm{H}), 5.05(\mathrm{sept}, \mathrm{J}=6.4 \mathrm{~Hz}, 1 \mathrm{H}), 3.77-3.75(\mathrm{~m}, 4 \mathrm{H}), 3.68(\mathrm{~s}, 2 \mathrm{H}), 2.57-2.54$ $(\mathrm{m}, 4 \mathrm{H}), 1.16(\mathrm{~d}, \mathrm{~J}=6.4 \mathrm{~Hz}, 6 \mathrm{H}) ;{ }^{31} \mathrm{P}$ NMR $\left(\mathrm{CDCl}_{3}\right) \delta 33.26 ;{ }^{13} \mathrm{C} \mathrm{NMR}\left(\mathrm{CDCl}_{3}\right) \delta 195.0$, $166.7,150.2$, 137.9, 136.7, 136.0, 135.2, 133.0, 131.8, 129.7, 128.5, 127.9, 127.1, 126.0, 
122.0, 66.9, 66.1, 64.2, 54.2, 21.5. HRMALDI: calcd for $\mathrm{C}_{38} \mathrm{H}_{37} \mathrm{~N}_{2} \mathrm{O}_{4} \mathrm{P}\left(\mathrm{M}+\mathrm{H}^{+}\right)$:

617.256, measured: 617.252 .

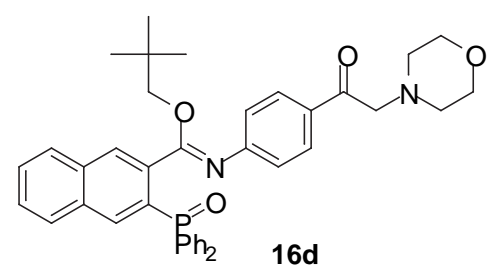

3-(Diphenyl-phosphinoyl)-N-[4-(2-morpholin-4-yl-acetyl)-phenyl]-naphthalene-2carboximidic acid 2,2-dimethyl-propyl ester (16d)

${ }^{1} \mathrm{H}$ NMR $\left(\mathrm{CDCl}_{3}\right) \delta 9.21(\mathrm{~s}, 1 \mathrm{H}), 8.30(\mathrm{~d}, \mathrm{~J}=8 \mathrm{~Hz}, 1 \mathrm{H}), 7.86-784(\mathrm{~m}, 2 \mathrm{H}), 7.76-7.70(\mathrm{~m}$, $4 \mathrm{H}), 7.64-7.32(\mathrm{~m}, 6 \mathrm{H}), 7.31(\mathrm{~d}, \mathrm{~J}=8.4 \mathrm{~Hz}, 2 \mathrm{H}), 7.24(\mathrm{dd}, \mathrm{J}=12.0,8.0 \mathrm{~Hz}, 2 \mathrm{H}), 7.05(\mathrm{~d}$, $\mathrm{J}=8.4 \mathrm{~Hz}, 2 \mathrm{H}), 3.77-3.75(\mathrm{~m}, 4 \mathrm{H}), 3.60(\mathrm{~s}, 2 \mathrm{H}), 3.58(\mathrm{~s}, 2 \mathrm{H}), 2.57-2.52(\mathrm{~m}, 4 \mathrm{H}), 0.79$ (s, $9 \mathrm{H}) ;{ }^{31} \mathrm{P} \mathrm{NMR}\left(\mathrm{CDCl}_{3}\right) \delta 33.18 ;{ }^{13} \mathrm{C} \mathrm{NMR}\left(\mathrm{CDCl}_{3}\right) \delta 195.0,166.5,150.3,137.9,136.7$, 136.0, 135.0, 132.9, 131.5, 129.6, 128.6, 128.0, 125.6, 121.8, 75.0, 67.1, 64.2, 54.2, 31.4, 26.5. HRMALDI: calcd for $\mathrm{C}_{40} \mathrm{H}_{41} \mathrm{~N}_{2} \mathrm{O}_{4} \mathrm{P}\left(\mathrm{M}+\mathrm{H}^{+}\right)$: 645.288, measured: 645.281.

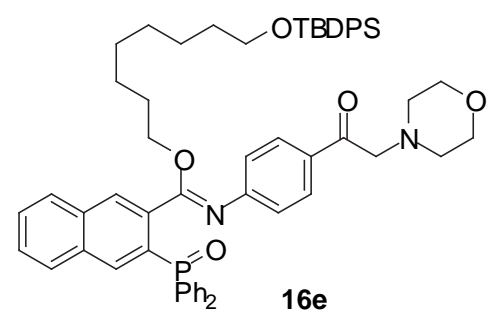

3-(Diphenyl-phosphinoyl)-N-[4-(2-morpholin-4-yl-acetyl)-phenyl]-naphthalene-2carboximidic acid 8-(tert-butyl-diphenyl-silanyoxy)-octyl ester (16e)

${ }^{1} \mathrm{H} \mathrm{NMR}\left(\mathrm{CDCl}_{3}\right) \delta 9.20(\mathrm{~s}, 1 \mathrm{H}), 8.30(\mathrm{~d}, \mathrm{~J}=8 \mathrm{~Hz}, 1 \mathrm{H}), 7.86-784(\mathrm{~m}, 2 \mathrm{H}), 7.76-7.70(\mathrm{~m}$, 4H), 7.64-7.32 (m, 16H), $7.31(\mathrm{~d}, \mathrm{~J}=8.4 \mathrm{~Hz}, 2 \mathrm{H}), 7.24(\mathrm{dd}, \mathrm{J}=12.0,8.0 \mathrm{~Hz}, 2 \mathrm{H}), 7.05$ (d, $\mathrm{J}=8.4 \mathrm{~Hz}, 2 \mathrm{H}), 3.80(\mathrm{t}, \mathrm{J}=6.8 \mathrm{~Hz}, 2 \mathrm{H}), 3.77-3.75(\mathrm{~m}, 4 \mathrm{H}), 3.68(\mathrm{~s}, 2 \mathrm{H}), 3.62(\mathrm{t}, \mathrm{J}=6.8$ $\mathrm{Hz}, 2 \mathrm{H}), 2.57-2.54(\mathrm{~m}, 4 \mathrm{H}), 1.59-1.49(\mathrm{~m}, 2 \mathrm{H}), 1.35-1.20(\mathrm{~m}, 4 \mathrm{H}), 1.17-1.09(\mathrm{~m}, 6 \mathrm{H})$, $1.02(\mathrm{~s}, 9 \mathrm{H}) ;{ }^{31} \mathrm{P} \mathrm{NMR}\left(\mathrm{CDCl}_{3}\right) \delta 33.23 ;{ }^{13} \mathrm{C} \mathrm{NMR}\left(\mathrm{CDCl}_{3}\right) \delta 166.5,150.3,137.9,136.7$, 136.0, 135.0, 132.9, 131.5, 129.6, 128.6, 128.0, 125.6, 121.8, 75.0, 35.5, 31.5, 34.6, 26.5, 22.7, 14.0. HRMALDI: calcd for $\mathrm{C}_{59} \mathrm{H}_{65} \mathrm{~N}_{2} \mathrm{O}_{5} \mathrm{PSi}\left(\mathrm{M}+\mathrm{H}^{+}\right)$: 941.447, measured: 941.432 .

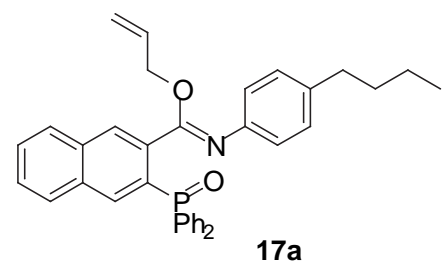

N-(4-Butyl-phenyl)-2-(diphenyl-phosphinoyl)-naphthalene-1-carboximidic acid allyl ester (17a)

${ }^{1} \mathrm{H}$ NMR $\left(\mathrm{CDCl}_{3}\right) \delta 9.17(\mathrm{~s}, 1 \mathrm{H}), 8.31(\mathrm{~d}, \mathrm{~J}=8 \mathrm{~Hz}, 1 \mathrm{H}), 7.87-784(\mathrm{~m}, 2 \mathrm{H}), 7.77-7.69(\mathrm{~m}$, $4 \mathrm{H}), 7.64-7.35(\mathrm{~m}, 6 \mathrm{H}), 7.31(\mathrm{~d}, \mathrm{~J}=8.4 \mathrm{~Hz}, 2 \mathrm{H}), 7.24(\mathrm{dd}, \mathrm{J}=12.0,8.0 \mathrm{~Hz}, 2 \mathrm{H}), 7.05(\mathrm{~d}$, $\mathrm{J}=8.4 \mathrm{~Hz}, 2 \mathrm{H}), 5.78(\mathrm{ddt}, \mathrm{J}=17.0,10.4,6.0 \mathrm{~Hz}, 1 \mathrm{H}), 5.21(\mathrm{dd}, \mathrm{J}=17.2,1.2 \mathrm{~Hz}, 1 \mathrm{H})$, $5.12(\mathrm{dd}, \mathrm{J}=10.4,1.2 \mathrm{~Hz}, 1 \mathrm{H}), 4.73(\mathrm{~d}, \mathrm{~J}=6.4 \mathrm{~Hz}, 2 \mathrm{H}), 2.55(\mathrm{t}, \mathrm{J}=7.6 \mathrm{~Hz}, 2 \mathrm{H}), 1.62-1.58$ 
(m, 2H), 1.38-1.31 (m, 2H), $0.94(\mathrm{t}, \mathrm{J}=7.43 \mathrm{H}) ;{ }^{31} \mathrm{P} \mathrm{NMR}\left(\mathrm{CDCl}_{3}\right) \delta 33.32 ;{ }^{13} \mathrm{C} \mathrm{NMR}$ $\left(\mathrm{CDCl}_{3}\right) \delta 166.9,151.2,137.9,137.5,136.7,135.0,134.2,133.0,131.8,131.5,130.4$, 129.6, 128.7, 128.0, 127.1, 125.9, 121.8, 115.1, 67.2, 35.5, 34.6, 22.7, 14.0. HRMALDI: calcd for $\mathrm{C}_{36} \mathrm{H}_{34} \mathrm{NO}_{2} \mathrm{P}\left(\mathrm{M}+\mathrm{H}^{+}\right)$: 544.240, measured: 544.235

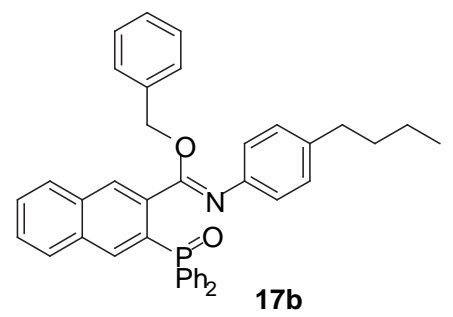

N-(4-Butyl-phenyl)-2-(diphenyl-phosphinoyl)-naphthalene-1-carboximidic acid benzyl ester (17b).

${ }^{1} \mathrm{H}$ NMR $\left(\mathrm{CDCl}_{3}\right) \delta 9.20(\mathrm{~s}, 1 \mathrm{H}), 8.30(\mathrm{~d}, \mathrm{~J}=8 \mathrm{~Hz}, 1 \mathrm{H}), 7.87-783(\mathrm{~m}, 2 \mathrm{H}), 7.75-7.70(\mathrm{~m}$, $4 \mathrm{H}), 7.63-7.22(\mathrm{~m}, 15 \mathrm{H}), 7.04(\mathrm{~d}, \mathrm{~J}=8.8 \mathrm{~Hz}, 2 \mathrm{H}), 4.65(\mathrm{~s}, 2 \mathrm{H}), 2.57(\mathrm{t}, \mathrm{J}=7.6 \mathrm{~Hz}, 2 \mathrm{H})$, $1.60-1.55(\mathrm{~m}, 2 \mathrm{H}), 1.40-1.32(\mathrm{~m}, 2 \mathrm{H}), 0.94(\mathrm{t}, \mathrm{J}=7.43 \mathrm{H}) ;{ }^{31} \mathrm{P} \mathrm{NMR}\left(\mathrm{CDCl}_{3}\right) \delta 33.22 ;{ }^{13} \mathrm{C}$ NMR $\left(\mathrm{CDCl}_{3}\right) \delta 166.2,150.4,140.6,137.9,136.6,135.1,134.2,132.9,131.5,130.4$, 129.6, 128.7, 128.6, 128.0, 127.3, 127.1, 125.9, 121.8, 67.9, 34.6, 33.5, 21.8, 13.8 . HRMALDI: calcd for $\mathrm{C}_{40} \mathrm{H}_{36} \mathrm{NO}_{2} \mathrm{P}\left(\mathrm{M}+\mathrm{H}^{+}\right)$: 594.256, measured: 594.251

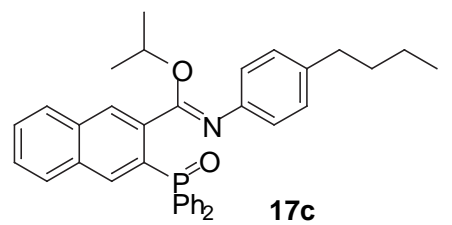

N-(4-Butyl-phenyl)-3-(diphenyl-phosphinoyl)-naphthalene-2-carboximidic acid isopropyl ester (17c)

${ }^{1} \mathrm{H}$ NMR $\left(\mathrm{CDCl}_{3}\right) \delta 9.20(\mathrm{~s}, 1 \mathrm{H}), 8.29(\mathrm{~d}, \mathrm{~J}=8 \mathrm{~Hz}, 1 \mathrm{H}), 7.86-784(\mathrm{~m}, 2 \mathrm{H}), 7.75-7.71(\mathrm{~m}$, 4H), 7.64-7.33 (m, 6H), $7.30(\mathrm{~d}, \mathrm{~J}=8.4 \mathrm{~Hz}, 2 \mathrm{H}), 7.25(\mathrm{dd}, \mathrm{J}=12.0,8.0 \mathrm{~Hz}, 2 \mathrm{H}), 7.05(\mathrm{~d}$, $\mathrm{J}=8.4 \mathrm{~Hz}, 2 \mathrm{H}), 5.05(\mathrm{sept}, \mathrm{J}=6.4 \mathrm{~Hz}, 1 \mathrm{H}), 2.55(\mathrm{t}, \mathrm{J}=7.6 \mathrm{~Hz}, 2 \mathrm{H}), 1.62-1.58(\mathrm{~m}, 2 \mathrm{H})$, $1.38-1.31(\mathrm{~m}, 2 \mathrm{H}), 1.16(\mathrm{~d}, \mathrm{~J}=6.4 \mathrm{~Hz}, 6 \mathrm{H}), 0.94(\mathrm{t}, \mathrm{J}=7.43 \mathrm{H}) ;{ }^{31} \mathrm{P} \mathrm{NMR}\left(\mathrm{CDCl}_{3}\right) \delta$ 33.26; ${ }^{13} \mathrm{C} \mathrm{NMR}\left(\mathrm{CDCl}_{3}\right) \delta 166.7,150.2,137.9,136.7,136.0,135.2,133.0,131.8,129.7$, 128.5, 127.9, 127.1, 126.0, 122.0, 66.9, 35.5, 34.6, 22.7, 21.5, 14.0. HRMALDI: calcd for $\mathrm{C}_{36} \mathrm{H}_{36} \mathrm{NO}_{2} \mathrm{P}\left(\mathrm{M}+\mathrm{H}^{+}\right)$: 546.256, measured: 546.249.

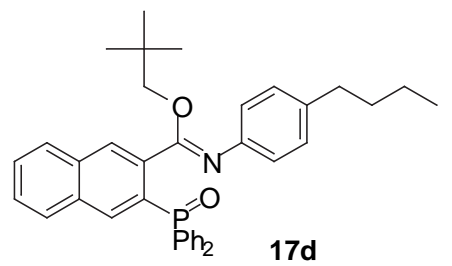

N-(4-Butyl-phenyl)-3-(diphenyl-phosphinoyl)-naphthalene-2-carboximidic acid 2,2dimethyl-propyl ester (17d)

${ }^{1} \mathrm{H} \mathrm{NMR}\left(\mathrm{CDCl}_{3}\right) \delta 9.21(\mathrm{~s}, 1 \mathrm{H}), 8.30(\mathrm{~d}, \mathrm{~J}=8 \mathrm{~Hz}, 1 \mathrm{H}), 7.86-784(\mathrm{~m}, 2 \mathrm{H}), 7.76-7.70(\mathrm{~m}$, $4 \mathrm{H}), 7.64-7.32(\mathrm{~m}, 6 \mathrm{H}), 7.31(\mathrm{~d}, \mathrm{~J}=8.4 \mathrm{~Hz}, 2 \mathrm{H}), 7.24(\mathrm{dd}, \mathrm{J}=12.0,8.0 \mathrm{~Hz}, 2 \mathrm{H}), 7.05(\mathrm{~d}$, $\mathrm{J}=8.4 \mathrm{~Hz}, 2 \mathrm{H}), 3.60(\mathrm{~s}, 2 \mathrm{H}), 2.55(\mathrm{t}, \mathrm{J}=7.6 \mathrm{~Hz}, 2 \mathrm{H}), 1.62-1.58(\mathrm{~m}, 2 \mathrm{H}), 1.38-1.31(\mathrm{~m}$, 
$2 \mathrm{H}), 0.94(\mathrm{t}, \mathrm{J}=7.43 \mathrm{H}), 0.79(\mathrm{~s}, 9 \mathrm{H}) ;{ }^{31} \mathrm{P} \mathrm{NMR}\left(\mathrm{CDCl}_{3}\right) \delta 33.18 ;{ }^{13} \mathrm{C} \mathrm{NMR}\left(\mathrm{CDCl}_{3}\right) \delta$ 166.5, 150.3, 137.9, 136.7, 136.0, 135.0, 132.9, 131.5, 129.6, 128.6, 128.0, 125.6, 121.8, 75.0, 35.5, 31.5, 34.6, 26.5, 22.7, 14.0. HRMALDI: calcd for $\mathrm{C}_{38} \mathrm{H}_{40} \mathrm{NO}_{2} \mathrm{P}\left(\mathrm{M}+\mathrm{H}^{+}\right)$: 574.287, measured: 574.265 .

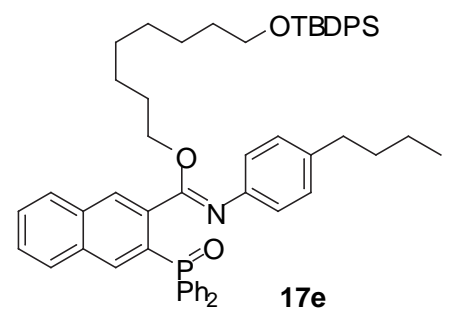

N-(4-Butyl-phenyl)-3-(diphenyl-phosphinoyl)-naphthalene-2-carboximidic acid 8(ter-butyl-diphenyl-silanyloxy)-octyl ester (17e)

${ }^{1} \mathrm{H} \mathrm{NMR}\left(\mathrm{CDCl}_{3}\right) \delta 9.20(\mathrm{~s}, 1 \mathrm{H}), 8.30(\mathrm{~d}, \mathrm{~J}=8 \mathrm{~Hz}, 1 \mathrm{H}), 7.86-784(\mathrm{~m}, 2 \mathrm{H}), 7.76-7.70(\mathrm{~m}$, 4H), 7.64-7.32 (m, 16H), 7.31 (d, J= 8.4 Hz, 2H), 7.24 (dd, J= 12.0, 8.0 Hz, 2H), 7.05 (d, $\mathrm{J}=8.4 \mathrm{~Hz}, 2 \mathrm{H}), 3.80(\mathrm{t}, \mathrm{J}=6.8 \mathrm{~Hz}, 2 \mathrm{H}), 3.62(\mathrm{t}, \mathrm{J}=6.8 \mathrm{~Hz}, 2 \mathrm{H}), 2.55(\mathrm{t}, \mathrm{J}=7.6 \mathrm{~Hz}, 2 \mathrm{H})$, 1.62-1.28 (m, 10H),1.17-1.09 (m,6H), $1.02(\mathrm{~s}, 9 \mathrm{H}), 0.94(\mathrm{t}, \mathrm{J}=7.4,3 \mathrm{H}) ;{ }^{31} \mathrm{P}$ NMR $\left(\mathrm{CDCl}_{3}\right) \delta 33.23 ;{ }^{13} \mathrm{C} \mathrm{NMR}\left(\mathrm{CDCl}_{3}\right) \delta 166.5,150.3,137.9,136.7,136.0,135.0,132.9$, 131.5, 129.6, 128.6, 128.0, 125.6, 121.8, 75.0, 35.5, 31.5, 34.6, 26.5, 22.7, 14.0. HRMALDI: calcd for $\mathrm{C}_{57} \mathrm{H}_{64} \mathrm{NO}_{3} \mathrm{PSi}\left(\mathrm{M}+\mathrm{H}^{+}\right)$: 870.447, measured: 870.443 . 


\section{INDEX OF HPLC TRACES}

HPLC 1: $\mathbf{6 a}+\mathrm{H}_{2} \mathrm{O}_{2}$ to generate phosphine oxide standard of $\mathbf{6 a}$

HPLC 2: Timetrial analysis of $\mathbf{6 a}$ coupling with azidoadenosine 7.

HPLC 3: Timetrial analysis of 6a coupling with 5'-azidoadenosine $2{ }^{\prime} 3^{\prime}$ isopropylidene.

HPLC 4: Timetrial analysis of $\mathbf{6 a}$ reaction with adenosine.

HPLC 5: Timetrial analysis of 6a coupling to 5'-triisopropylsilyl C8-azidoadenosine 


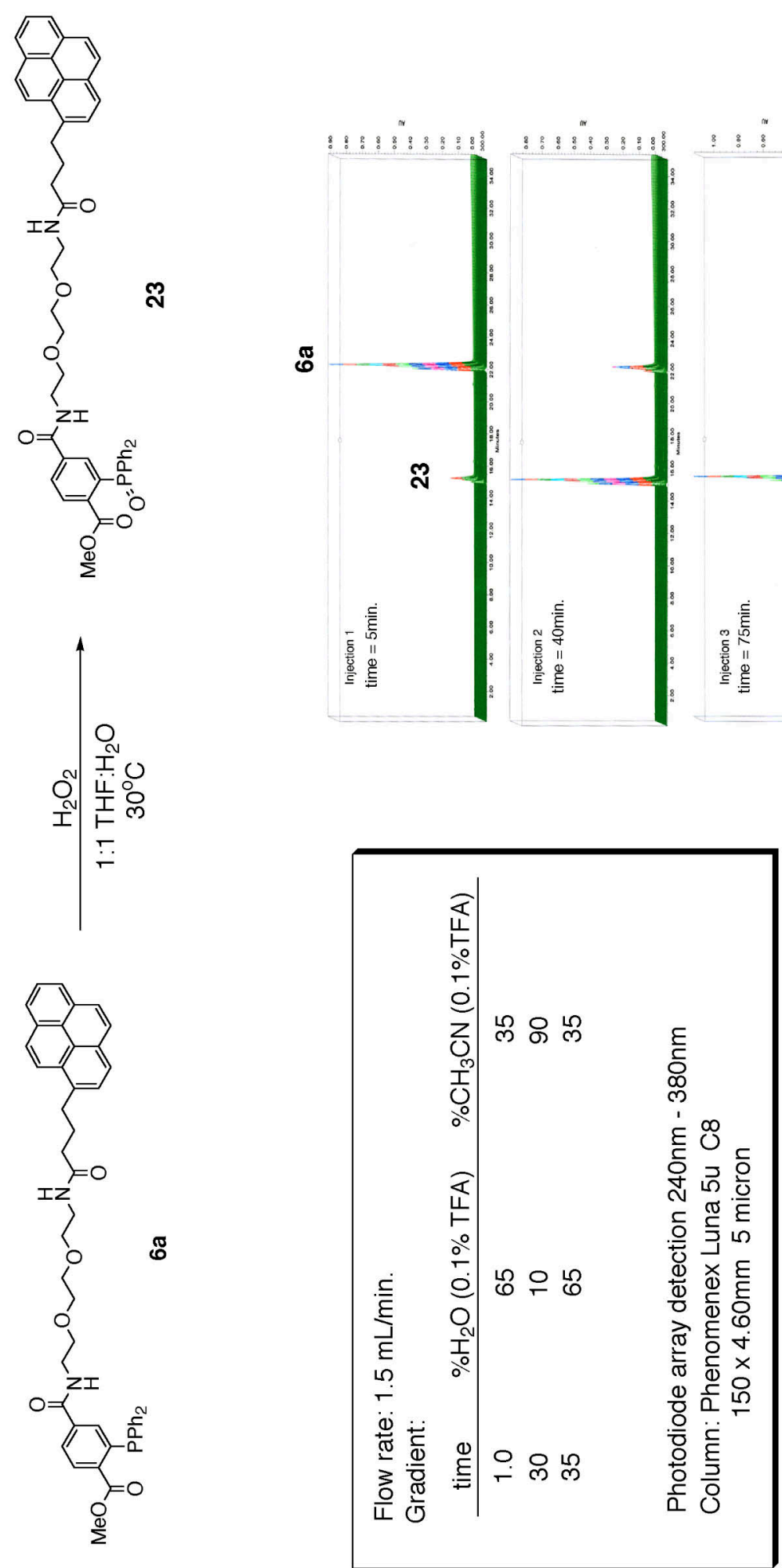


HPLC 2
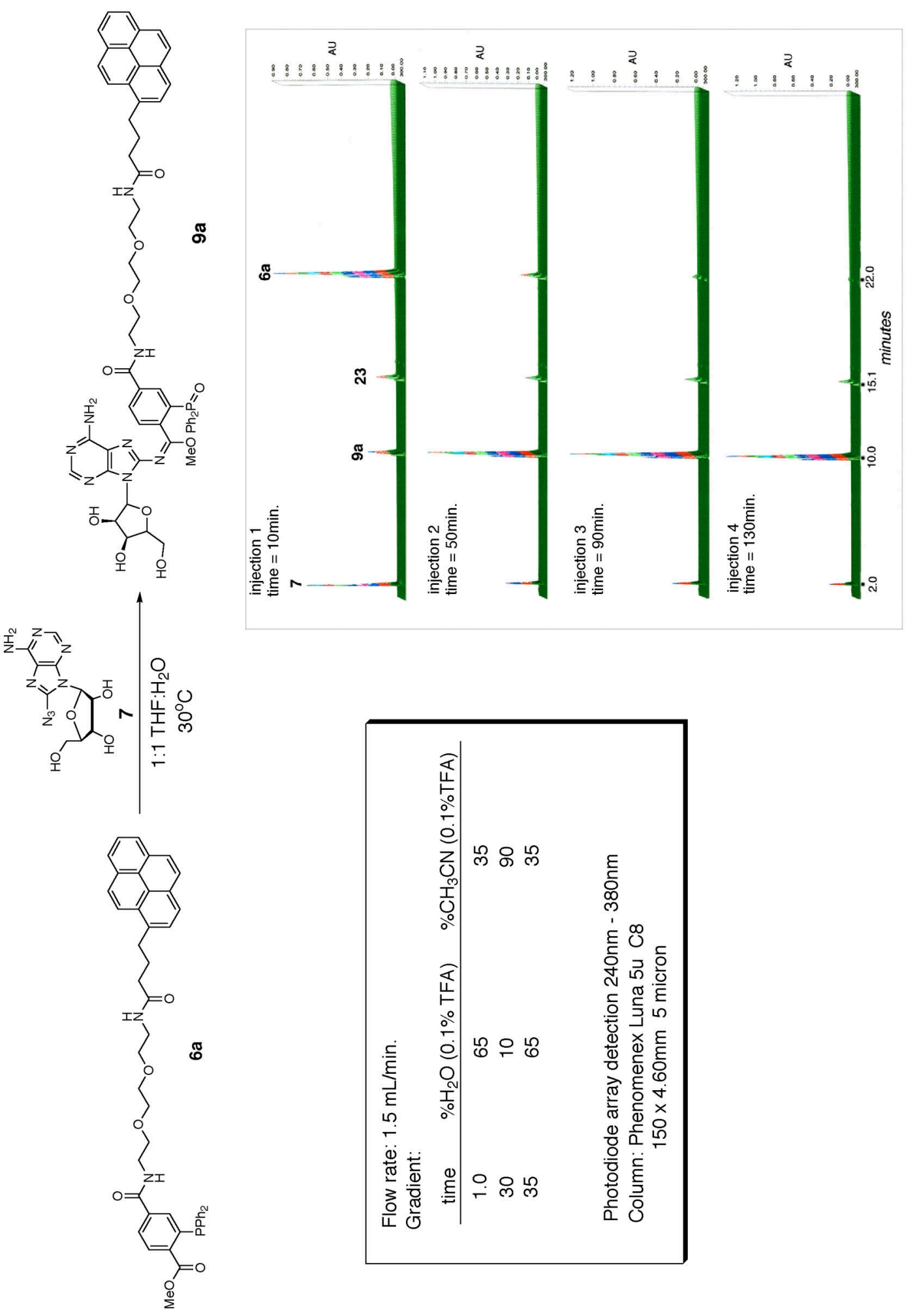
HPLC 3
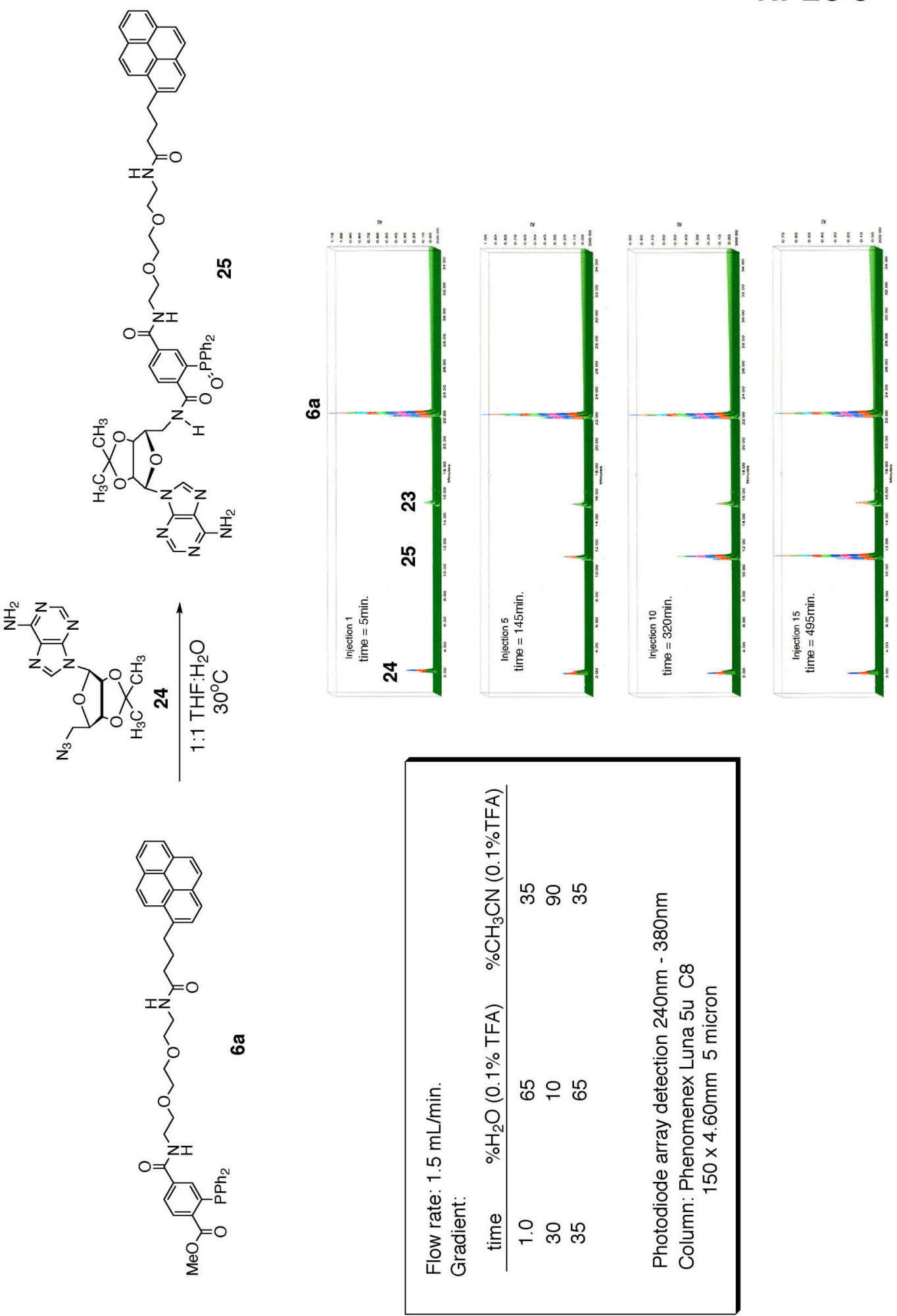
HPLC 5
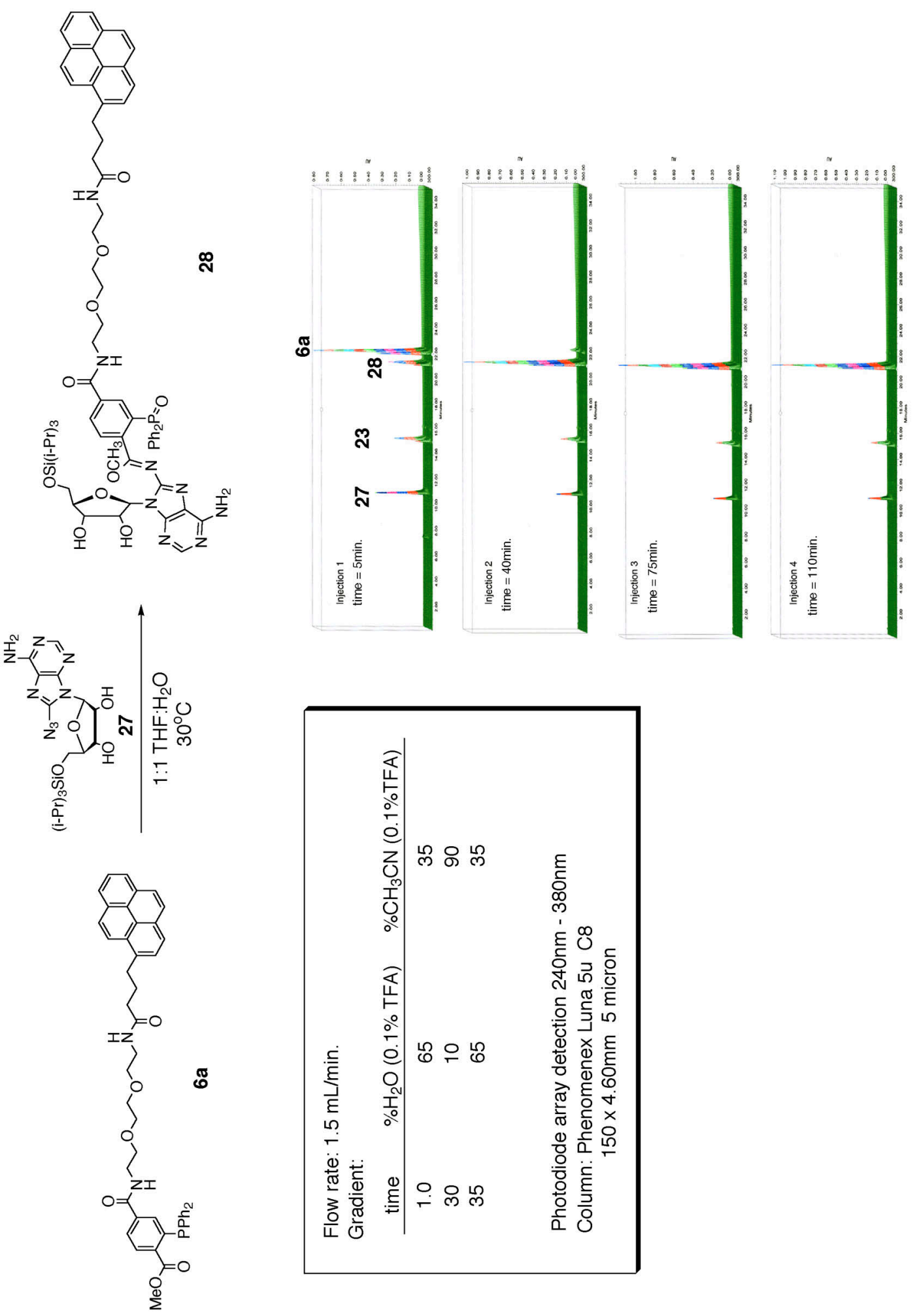